\title{
Aves de um remanescente florestal do Quadrilátero Ferrífero, Minas Gerais
}

\author{
Jordana Demicheli Ferreira ${ }^{1,2}$, Lílian Mariana Costa $^{1} \&$ Marcos Rodrigues $^{1}$ \\ ${ }^{1}$ Laboratório de Ornitologia, Departamento de Zoologia, \\ Instituto de Ciências Biológicas - ICB, Universidade Federal de Minas Gerais - UFMG \\ CP 486, CEP 31270-901, Belo Horizonte, MG, Brasil, www.icb.ufmg.br \\ ${ }^{2}$ Autor para correspondência: Jordana Demicheli Ferreira, e-mail: jdemicheli@ gmail.com
}

FERREIRA, J.D., COSTA, L.M. \& RODRIGUES, M. Birds of a forest remnant in the Iron Quadrangle of Minas Gerais, southeastern Brazil. Biota Neotrop. 9(3): http://www.biotaneotropica.org.br/v9n3/en/ abstract?article+bn00509032009.

\begin{abstract}
The Iron Quadrangle (IQ) is a region of southeastern Brazil known for having good-quality mineral deposits of worldwide economic importance. The iron extraction process causes large scale landscape transformations with unknown impact on the local biodiversity. This paper presents a bird list of a remnant forest fragment in the IQ. The surveyed area named "Mata Samuel de Paula" is a 147 ha conservation unit located in a transitional area between two large biomes: the Atlantic Forest and the Cerrado. Its vegetation is a mix of second-growth semi-deciduous forest, scrublands, and ferruginous rocky fields. Bird censuses were carried out during a one year period using observational transects and mist-net captures. A total of 159 species were recorded and richness was estimated to be about 188 species. Information on habitat, frequency of occurrence and seasonality, migratory, endemic, and conservation status were also obtained for each species. Four species recorded are threatened; twenty-eight species are endemic to the Atlantic Forest, three to the Cerrado, and one to particular mountain tops in southeastern Brazil. An analysis of bird lists from five other protected areas in IQ suggests that additional 50 endemic and 21 threatened species may exist in the region. Multiple regression showed a strong relationship between number of Atlantic Forest endemic species, area and longitude of natural reserves, a result also suggested by comparing the number of threatened species with the area of the reserves through simple regression. Results also indicate that a number of the threatened species are restricted to bigger reserves. The IQ is a valuable region for avian conservation efforts, but faces significant disturbance pressures from highly antagonistic economic interests. Despite the enormous threats the region faces, there are still large gaps in the baseline information yielded by biodiversity surveys, which is a basic tool for implementing effective conservation plans.
\end{abstract}

Keywords: Atlantic Forest, bird communities, Cerrado, mining activities.

FERREIRA, J.D., COSTA, L. M. \& RODRIGUES, M. Aves de um remanescente florestal do Quadrilátero Ferrífero, Minas Gerais. Biota Neotrop. 9(3): http://www.biotaneotropica.org.br/v9n3/pt/ abstract?article+bn00509032009.

Resumo: O Quadrilátero Ferrífero (QF), localizado em Minas Gerais, é líder mundial na produção de minerais metálicos, principalmente minério de ferro. Essa atividade mineradora é responsável pela modificação da paisagem com impactos ainda pouco estudados sobre a biodiversidade regional. O presente trabalho apresenta a lista de aves de um remanescente florestal da região, a Reserva Particular do Patrimônio Natural Mata Samuel de Paula. A reserva possui 147 ha localizados em área de transição entre a Mata Atlântica e o Cerrado, no município de Nova Lima. Sua vegetação é composta por floresta estacional semidecídua secundária, fitofisionomias campestres de cerrado e campos ferruginosos. O levantamento foi feito por observação em transecções e captura com redes ao longo de um ano. Registraram-se 159 espécies de aves, das quais foram avaliados hábitat e frequiência de ocorrência, sazonalidade e situação de migração, endemismo e conservação. A riqueza foi estimada em 188 espécies. A lista apresenta quatro espécies ameaçadas de extinção, além de 28 espécies endêmicas da Mata Atlântica, três do Cerrado e uma dos topos de montanha do sudeste do Brasil. A análise de inventários de outras cinco Unidades de Conservação do QF mostrou mais 50 espécies endêmicas, além de outras 20 ameaçadas de extinção. Encontrou-se forte relação entre o número de espécies endêmicas da Mata Atlântica, área e longitude das reservas, por regressão múltipla $\left(R^{2}=0,91 ; p=0,03\right)$, e entre o número de espécies ameaçadas e a área das reservas, por regressão simples $\left(R^{2}=0,84 ; p=0,01\right)$. Constatou-se ainda que grande parte das espécies ameaçadas é encontrada somente nas maiores reservas. A preservação do QF está ameaçada por fortes interesses econômicos e, apesar da destruição de habitat oriunda da exploração de suas qualidades minerais e da urbanização, existe carência de estudos sobre sua biodiversidade, ferramentas básicas para o planejamento de um sistema de reservas eficiente.

Palavras-chave: Mata Atlântica, campos ferruginosos, Cerrado, mineração. 


\section{Introdução}

O Quadrilátero Ferrífero (QF) está situado inteiramente no estado de Minas Gerais, sudeste do Brasil. A área cobre aproximadamente $7.200 \mathrm{~km}^{2}$ e está localizada entre a Mata Atlântica e o Cerrado (Jacobi et al. 2007), biomas considerados hotspots (Myers et al. 2000). A situação dos biomas é refletida em sua avifauna, que possui os maiores números de espécies endêmicas ameaçadas de extinção no Brasil (54 espécies na Mata Atlântica e 14 no Cerrado) (Marini \& Garcia 2005). A Mata Atlântica foi drasticamente reduzida pela acelerada degradação antrópica, que se instaura com o início da colonização do Brasil (Dean 2002). O Cerrado brasileiro também sofre acentuada redução e as estimativas indicam que aproximadamente $80 \%$ de sua vegetação original já foram alterados devido, principalmente, a atividades de pecuária e agricultura (Myers et al. 2000). O QF é líder mundial na produção de minerais metálicos, principalmente o minério de ferro, o que resulta em intensa modificação da paisagem com impactos ainda pouco estudados sobre a biodiversidade regional (Jacobi et al. 2007, Jacobi \& Carmo 2008). A região também sofre grande pressão pela expansão urbana, desmatamento e turismo e é classificada como de importância biológica especial por apresentar endemismos de anfíbios e plantas, alta riqueza de vertebrados e um ambiente único no estado, os campos ferruginosos (Drummond et al. 2005).

Em decorrência das qualidades do solo para extração de minério, grande parte das terras e dos remanescentes de Mata Atlântica e Cerrado da região pertence a empresas mineradoras (Figueredo \& Salino 2005). No município de Nova Lima, a especulação imobiliária fez do loteamento de áreas onde não há interesse em minerar uma importante fonte de lucro para essas empresas (Figueredo \& Salino 2005). Em decorrência, é notável o grande e crescente número de condomínios residenciais no município, que atendem principalmente à expansão de Belo Horizonte (Souza \& Brito 2006).

$\mathrm{O}$ alto grau de ameaça que existe nos biomas Mata Atlântica e Cerrado, especificamente na região do Quadrilátero Ferrífero, torna urgente a realização de pesquisas para ampliar os conhecimentos sobre esse ambiente e sua comunidade biótica, sendo o procedimento preliminar o levantamento de espécies (Drummond et al. 2005). Apresenta-se nesse trabalho uma lista inédita das aves que ocorrem em um dos fragmentos florestais remanescentes do Quadrilátero Ferrífero, a Reserva Particular do Patrimônio Natural (RPPN) Mata Samuel de Paula (doravante MSP). Além disso, foi adotada uma abordagem comparativa das espécies ameaçadas e endêmicas da MSP e de outras cinco Unidades de Conservação (UC's) do Quadrilátero Ferrífero.

\section{Material e Métodos}

A MSP, RPPN implantada no ano 2000, é um fragmento florestal que pertence à empresa de mineração AngloGold Ashanti. A reserva possui 147,83 ha e está inserida na transição entre Mata Atlântica e Cerrado, na porção norte do Quadrilátero Ferrífero (20 $03^{\circ} 30^{\prime}$ ' S e $43^{\circ} 52$ ' 25 ' W), com altitudes entre 810 e $1.200 \mathrm{~m}$, no município de Nova Lima, MG (Figura 1). A região apresenta precipitação total anual entre 1.000 e $1.550 \mathrm{~mm}$ e temperatura média anual de $18^{\circ} \mathrm{C}$, com verões chuvosos e invernos brandos e secos. A umidade relativa média anual é de $80 \%$, sendo os meses mais úmidos dezembro e janeiro e, os mais secos, agosto e setembro (Figueredo \& Salino 2005).

A maior parte do entorno da MSP é constituída por condomínios residenciais, fazendo fronteira também com áreas urbanizadas da cidade de Nova Lima. O ribeirão dos Cristais determina um dos limites da RPPN e desemboca no rio das Velhas, afluente do Rio São Francisco
A exploração em Nova Lima teve início no final do século XVII com a chegada de um grupo de bandeirantes, liderados por Domingos Rodrigues da Fonseca Leme, que ali encontraram ouro e iniciaram o garimpo (Vasconcelos 1999). A região era coberta por espessas matas e o ribeirão dos Cristais foi um dos primeiros locais de extração aurífera. Durante o século XIX, o ribeirão dos Cristais continuou sendo o principal curso de água usado pela Mineração Morro Velho, que durante muito tempo foi a principal jazida aurífera do planeta (Burton 1869).

Praticamente toda a mata da MSP foi outrora derrubada, sendo a maior parte da vegetação constituída atualmente de floresta estacional semidecidual em diversos estágios de sucessão. Existe também uma pequena mancha de Cerrado e nas partes mais altas e de acentuada declividade predominam os campos ferruginosos, que possuem formação herbácea e arbustiva característica desenvolvida sobre a canga (Jacobi et al. 2007). Além dessas fitofisionomias, existem áreas de vegetação fortemente alteradas pelo homem (antigas pastagens e plantações). A reserva está situada a menos de $2 \mathrm{~km}$ da Mata do Jambreiro, RPPN de 902 ha, separada da primeira por uma faixa intensamente urbanizada, incluindo uma rodovia estadual.

O levantamento de aves da RPPN MSP teve início em 14/09/05 e se estendeu até 19/09/06. Durante esse período foram realizadas 13 campanhas na reserva, totalizando 236 horas de campo em 33 dias de levantamento. As amostragens se concentraram diariamente entre 5:00 e 11:00 horas e entre 15:00 e 19:00 horas.

Os dados foram coletados através de dois métodos: audiovisual e captura em redes de neblina. O método audiovisual foi realizado utilizando trilhas e estradas pré-existentes, onde se caminhou anotando-se as espécies de aves observadas e ouvidas, com o auxílio de binóculos (Nikon 8X40, 8.2 ${ }^{\circ}$ ). Eventualmente foram feitas incursões na mata onde não há trilhas. Espécies crípticas e não identificadas em campo tiveram suas vocalizações registradas por gravadores (Sony TCM5000EV e HI-MD MZ-RH910) acoplados a microfone unidirecional (Sennheiser ME-66). Posteriormente, acervos de vocalizações de aves foram consultados para a confirmação da identidade dessas espécies (Gonzaga \& Castiglioni 2001, Vielliard 1995, 2002, Xeno-canto 2008). Algumas gravações foram utilizadas para realizar playback, que consiste na reprodução de vocalizações com a finalidade de atrair indivíduos não identificados e assim possibilitar sua identificação.

As capturas foram realizadas por meio de redes de neblina (de $12 \times 2,5 \mathrm{~m}$, com malha de $36 \mathrm{~mm}$ ) armadas em trilhas estreitas e concentradas na área de mata. As aves capturadas foram marcadas com anilhas metálicas numeradas cedidas pelo Centro Nacional de Pesquisa e Conservação de Aves Silvestres do IBAMA (CEMAVE), o que permitiu excluir as recapturas das contagens de abundância de capturas. Além disso, algumas aves foram fotografadas com câmeras digitais (Sony DSC-S40, Canon PowerShot A300 e HP Photosmart 735), o que também possibilitou a confirmação de alguns registros. O esforço de captura totalizou 429 horas-rede.

A lista de espécies foi elaborada seguindo ordem sistemática e nomenclatura sugeridas pelo Comitê Brasileiro de Registros Ornitológicos - CBRO (2008). A situação de endemismo das aves registradas foi extraída de Stotz et al. (1996), Silva \& Bates (2002) e Vasconcelos et al. (2003a) e a de conservação seguiu as listas de BirdLife International (2000, 2008) e de Machado et al. (1998).

O cálculo da riqueza de espécies por amostragem é dificultado devido à possibilidade de várias espécies não serem registradas durante o esforço amostral, que influencia no número de espécies detectado. O uso de estimadores é aconselhado em inventários dos quais a curva de acumulação de espécies não atingiu uma assíntota óbvia (Colwell $\&$ Coddington 1994). Com o intuito de reduzir o efeito do tamanho do esforço na riqueza de espécies e torná-la, desse modo, mais comparável a de outras áreas (Smith \& Van Belle 1984), foi realizada uma 


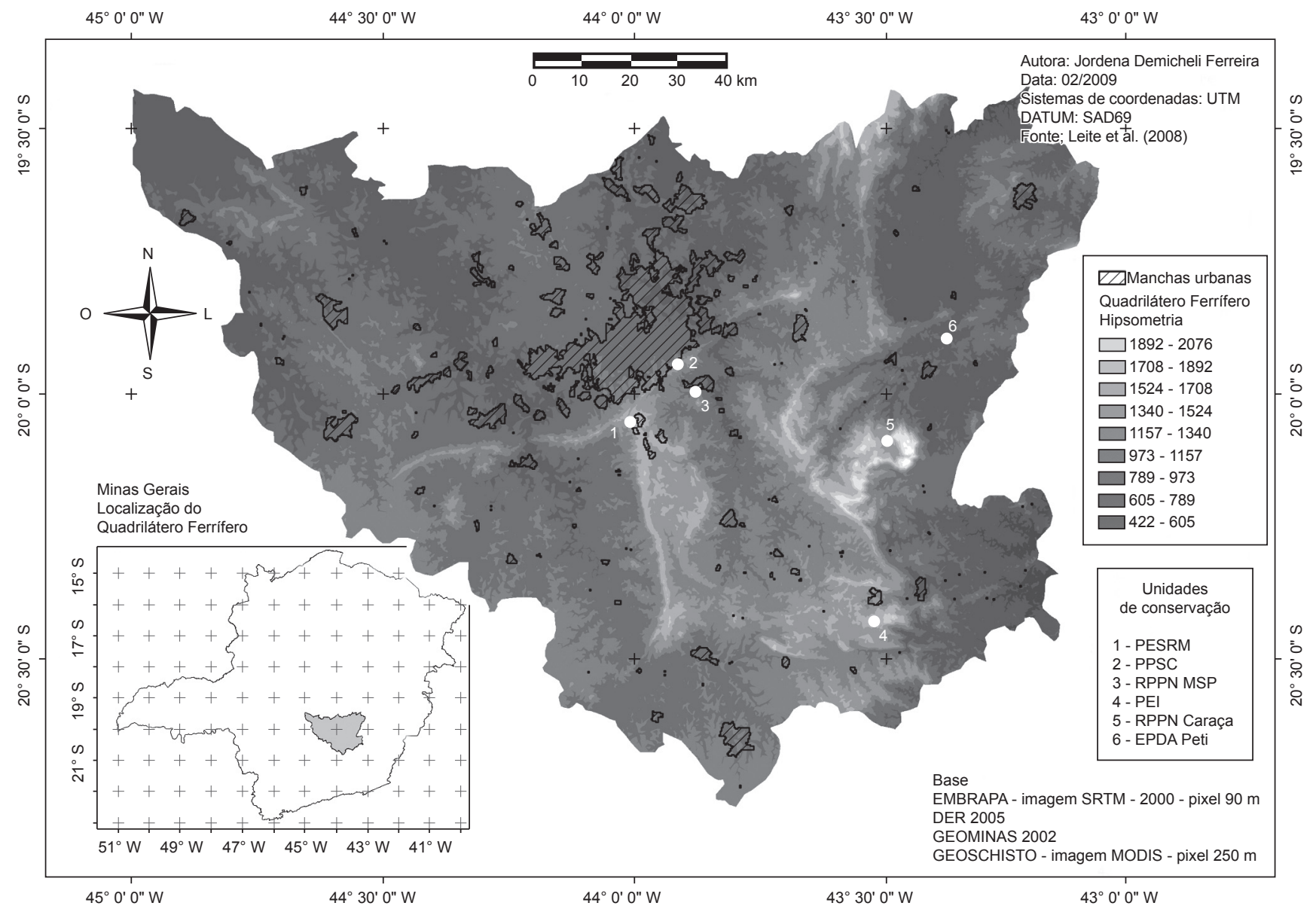

Figura 1. Localização do Quadrilátero Ferrífero, da área de estudo (RPPN MSP) e das outras cinco unidades de conservação analisadas.

Figure 1. Localization of the Iron Quadrangle region, surveyed area (RPPN MSP) and others five conservation units examined.

estimativa da riqueza de espécies de aves da MSP. Os cálculos foram realizados utilizando-se o estimador "Jackknife de primeira-ordem", por meio do programa "EstimateS" (Rodrigues et al. 2005, Rodrigues \& Michellin 2005, Colwell 2006). O método usa a acumulação das espécies observadas por amostras para realizar a estimativa da riqueza baseada no número de espécies perdidas quando amostras são removidas, sendo a estimativa, conseqüentemente, uma função das espécies presentes em somente uma amostra (Heltshe \& Forrester 1983, Smith \& Van Belle 1984). A unidade amostral utilizada foi cada dia de levantamento. A variância da riqueza estimada fornece o intervalo de confiança de $95 \%$ (Heltshe \& Forrester 1983).

A frequiência de ocorrência de uma espécie foi definida segundo Rodrigues et al. (2005) como "abundante" quando registrada em 75 a $100 \%$ das visitas à área; "comum" quando registrada em 50 a 74\% das visitas; "escassa" quando detectada em 25 a $49 \%$ das visitas. A espécie foi considerada "rara" quando registrada em menos de $24 \%$ das visitas, a não ser se observada apenas uma vez, quando foi considerada "ocasional". Os ambientes em que as espécies ocorrem na reserva foram discriminados como "área aberta degradada", "mata", "cerrado" e "campos ferruginosos".

A sazonalidade da comunidade de aves na MSP foi avaliada considerando as estações do ano em que cada uma ocorreu na reserva. As espécies foram ainda classificadas quanto à condição migratória, segundo informações extraídas de Chesser (1994) e Stotz et al. (1996): "migrantes austrais" são aqueles que se reproduzem na América do Sul continental e temperada, mas migram para o norte no inverno austral (Chesser 1994); "migrantes neárticos", são os que não se reproduzem nos trópicos; "migrantes neárticos parciais", são os que migram para sul no inverno, porém somente para lugares em que há populações estabelecidas que lá se reproduzam; e "migrantes regionais", os que realizam migrações sazonais de menor escala (Stotz et al. 1996).

Realizou-se uma análise comparativa das espécies endêmicas e/ou ameaçadas presentes na MSP e em outras cinco Unidades de Conservação do Quadrilátero Ferrífero: Parque Estadual do Itacolomi (PEI, 7.543 ha), Reserva Particular do Patrimônio Natural do Caraça (RPPN Caraça, 11.233 ha), Parque Paredão da Serra do Curral (PPSC, 39 ha), Estação de Pesquisa e Desenvolvimento Ambiental de Peti (EPDA Peti, 605 ha) e Parque Estadual Serra do Rola-Moca (PESRM, 3.941 ha) (Figura 1). As comparações foram realizadas a partir de consulta às listas de Ribon (2006), Vasconcelos \& Melo-Júnior. (2001) e Vasconcelos et al. (2003b), Vasconcelos (2007a), Faria et al. (2006), Vasconcelos (2007b) e Hass et al. (2005). O inventário do PESRM é advindo de uma avaliação ecológica rápida realizada em nove dias consecutivos, para o plano de manejo do parque. A lista de espécies de aves do PEI também é referente a uma avaliação ecológica rápida feita para o plano de manejo; porém, foi também contemplado nessa lista um inventário feito na década de 80 e muitos registros 
não-sistemáticos, o que certamente tornou a lista mais fidedigna. A lista de aves do Caraça é derivada de levantamento que abrangeram vários anos e que inclui e discute os registros realizados por Carnevalli (1980). A lista apresentada para o PPSC é oriunda de observações não sistemáticas feitas de 1993 a 2004 e a lista de Peti é provinda de levantamentos que remontam à década de 80 (Carnevalli et al. 1989) e inventário mais recente com amostras mensais por quase três anos (Faria et al. 2006).

Foi realizada uma regressão linear simples para testar se o aumento do número de espécies ameaçadas está relacionado ao aumento da área das Unidades de Conservação (UC's). Além disso, foi avaliado por regressão linear múltipla se o número de espécies endêmicas da Mata Atlântica é influenciado pela área das UC e pela longitude de sua localização. A variável longitude é dada pela diferença entre as coordenadas ' $x$ ' das cinco reservas mais a leste e a coordenada ' $x$ ' da reserva mais a oeste, em UTM (Universal Transversa de Mercator). As regressões foram desenvolvidas com o auxílio do pacote estatístico BioEstat 5.0 (Ayres et al. 2007), sendo realizada análise dos resíduos para avaliar a homogeneidade da variância e normalidade dos escores de Y, a linearidade dos valores observados e a presença de valores externos (Sokal \& Rolhf 1995).

\section{Resultados}

Foram registradas 159 espécies de aves na MSP, pertencentes a 42 famílias (Anexo 1). Dezoito famílias são de aves não-Passeriformes e 24 de Passeriformes. As famílias melhor representadas são: Tyrannidae (37 espécies), Furnariidae e Thraupidae (11 cada) e Emberizidae e Trochilidae (10 cada). A curva do coletor não atingiu uma assíntota óbvia e a riqueza estimada de espécies para a RPPN foi de 188,06 \pm 7,09 espécies (Figura 2).

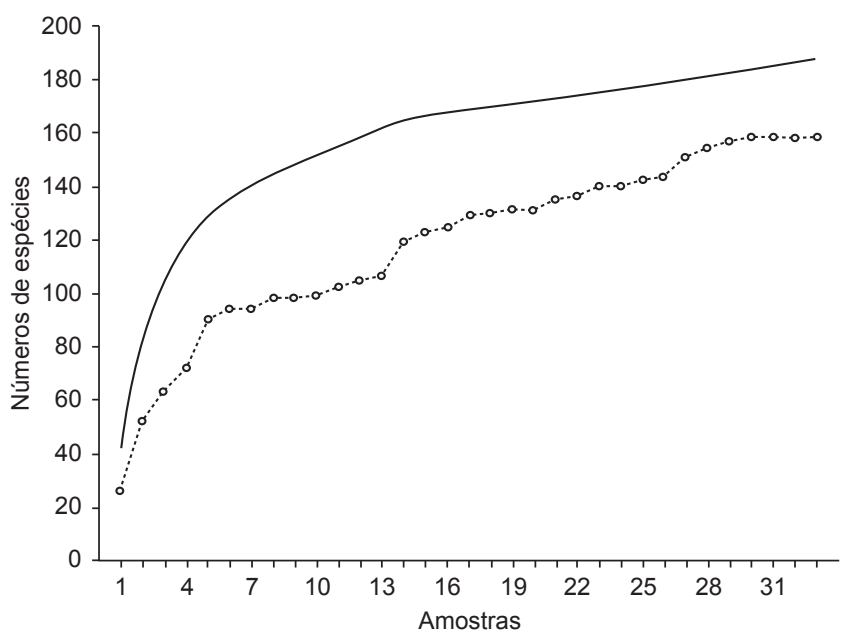

Figura 2. Curva do coletor observada (linha tracejada) mostrando o acúmulo de espécies registradas na RPPN Mata Samuel de Paula (MG) ao longo das amostragens. Total estimado da riqueza de espécies obtido pelo método Jackknife (linha cheia), baseado em números sucessivamente maiores de amostras, onde cada ponto estimado é a média das riquezas obtidas por aleatorização da ordem de acumulação das amostras.

Figure 2. Observed collector curve (sketched line) showing the accumulation of species registered at RPPN Mata Samuel de Paula (MG) along the samples. Estimated total species richness by the Jackknife method based on successively larger numbers of samples from the data set (thick line), in witch each point is the mean of estimates based on randomization of sample accumulation order.
Foram capturados 141 indivíduos de 45 espécies, pertencentes a 17 famílias de aves. As espécies que tiveram maior frequiência de indivíduos capturados foram Pyriglena leucoptera (Vieillot, 1818) (12 indivíduos), Basileuterus hypoleucus Bonaparte, 1830 (11), Conopophaga lineata (Wied, 1831) e Trichothraupis melanops (Vieillot, 1818) (10 indivíduos cada). A família que teve o maior número de espécies capturadas foi Tyrannidae (21 espécies), seguida de Thamnophilidae e Thraupidae (19 espécies cada) e de Parulidae (18). Foi capturado um individuo de Neopelma pallescens (Lafresnaye, 1983), registro raro no Quadrilátero Ferrífero. Outro registro da espécie na região foi realizado em EPDA Peti por Faria et al. (2006), onde também foram registradas Neopelma chrysocephalum (Pelzeln, 1868) por Vasconcelos (2007b). Neopelma aurifrons (Wied, 1831) foi registrada em Peti somente por Carnevalli et al. (1989) antes de a espécie ser revisada por Whitney et al. (1995), que provaram serem as subespécies $N$. a. aurifrons e $N$. a. chrysocephalum espécies distintas (registro revisado por Vasconcelos 2007b). A RPPN Caraça possui registro recente de $N$. chrysocephalum (Pelzeln, 1868) por Vasconcelos e Melo-Júnior (2001), enquanto que $N$. pallescens foi registrada nessa reserva somente por Ney Carnevalli no levantamento realizado em 1980 (Vasconcelos e Melo-Júnior 2001). Em outras áreas do Quadrilátero Ferrífero (PEI, PPSC e PESRM) nenhuma espécie desse gênero foi registrada.

Dezoito espécies mostraram-se abundantes, 14 comuns, 35 escassas, 63 raras e 29 ocasionais. Cento e trinta e sete espécies foram registradas exclusivamente em um ambiente da reserva; sendo 83 espécies no ambiente "mata", 22 em "área aberta degradada", 26 em "cerrado" e seis em "campos ferruginosos". Outras 22 espécies foram encontradas em mais de um hábitat discriminado.

Trinta e sete por cento das espécies foram registradas em todas as estações do ano, enquanto $26 \%$ foram detectadas somente em uma estação; $20 \%$ em duas e $16 \%$ em três. Um considerável número de espécies foi registrado somente em uma estação e na primavera foi quando houve o maior número de espécies detectadas (Tabela 1).

São conhecidos movimentos de migração para 41 espécies registradas na MSP (26\% do total). A maior parte dessas espécies é migrante austral (34). Cinco são migrantes neárticas parciais, uma migrante neártica e outra migrante em escala regional. Algumas das espécies realizam mais de um tipo de migração, sendo consideradas tanto migrantes austrais como neotropicais (seis espécies). Enquanto grande parte das espécies residentes foi registrada em todas as estações (50 espécies compondo $42 \%$ das residentes), somente $24 \%$ das migratórias (10 espécies) foram registradas em todas as estações. Setenta e seis por cento das espécies migratórias não foram detectadas em todas as estações na MSP. Alguns exemplos de espécies registradas nessa reserva que realizam movimentos migratórios estão dispostos abaixo:

Vireo olivaceus (Linnaeus, 1766) (Vireonidae). Espécie migrante do neártico, que não se reproduz nos trópicos. Sua área de invernada é inteiramente ou quase que inteiramente neotropical. Vireo olivaceus

Tabela 1. Número de espécies registradas exclusivamente em uma estação do ano e número total de espécies observadas em cada estação.

Table 1. Number of species recorded exclusively in one season and total number of species detected in each season.

\begin{tabular}{lcccc}
\hline \multicolumn{1}{c}{$\begin{array}{c}\text { Número de } \\
\text { espécies }\end{array}$} & Verão & Outono & Inverno & Primavera \\
\cline { 2 - 5 } & 6 & 7 & 9 & 19 \\
\hline $\begin{array}{l}\text { Exclusivas } \\
\text { da estação }\end{array}$ & & 101 & 108 & 117 \\
Total da estação & 99 & & & \\
\hline
\end{tabular}


também é categorizado como migrante austral (Stotz et al. 1996). Na MSP, a espécie foi registrada por gravação e captura de agosto a janeiro.

Turdus subalaris (Seebohm, 1887) (Turdidae). Espécie migrante austral. Possui população cisandina migratória, para qual há relatos de reprodução em São Paulo, Rio Grande do Sul, Argentina e fronteira paraguaia. As áreas de invernada da população migrante são a borda sul da Floresta Amazônica, porém suas rotas de migração são desconhecidas. Movimenta-se apenas na mata ciliar, onde se alimenta de frutos (Alves 2007). Um indivíduo de T. subalaris foi registrado por observação em 14/09/05 na mata ciliar do ribeirão dos Cristais. Em Minas Gerais há outros registros entre maio e setembro (ver Alves 2007).

Haplospiza unicolor Cabanis, 1851 (Emberizidae). Espécie endêmica da Mata Atlântica que realiza movimentos regionais em função da disponibilidade de recurso (Stotz et al. 1996, Vasconcelos et al. 2005). Essa espécie é dependente de sementes de bambu e desloca-se por grandes distâncias entre pontos de floração ou frutificação dessas gramíneas (Olmos 1996). Somente um indivíduo foi registrado por gravação apenas no dia 20/07/06, no interior da mata.

Foram registradas na MSP 28 espécies endêmicas da Mata Atlântica, três espécies endêmicas do Cerrado (tapaculo-de-colarinho Melanopareia torquata (Wied, 1831), gralha-do-campo Cyanocorax cristatellus (Temminck, 1823) e capacetinho-do-oco-do-pau Poospiza cinerea Bonaparte, 1850) e uma espécie endêmica dos topos de montanhas do sudeste do Brasil (rabo-mole-da-serra Embernagra longicauda Strickland, 1844) (Anexo 1). Melanopareia torquata (Melanopareiidae) foi freqüentemente registrada nas áreas de campos ferruginosos da MSP. A vocalização de C. cristatellus (Corvidae) foi escutada e gravada várias vezes na MSP, nas bordas da mata. Essa espécie tem sido freqüentemente observada em áreas desmatadas e está expandindo sua área de vida para locais onde a floresta foi substituída por áreas abertas (Lopes 2008). Quatro das espécies endêmicas constam em listas de espécies ameaçadas de extinção (ver abaixo):

Campephilus robustus (Lichtenstein, 1818) (Picidae). Também conhecida popularmente como pica-pau-rei, essa espécie é endêmica da Mata Atlântica e considerada em perigo de extinção em Minas Gerais pela acelerada destruição de seu hábitat, alto grau de dependência de ambientes florestais e por possuir populações isoladas. $\mathrm{O}$ desmatamento é a maior ameaça para essa espécie, que necessita de árvores de grande porte, mais velhas e de troncos grossos para a reprodução (Machado et al. 1998). O pica-pau-rei foi visto e gravado em 14/02/06.

Scytalopus indigoticus (Wied, 1831) (Rhinocryptidae). Essa espécie, conhecida como macuquinho, é endêmica da Mata Atlântica e considerada globalmente quase-ameaçada de extinção (BirdLife International 2000, 2008). A espécie foi registrada em três locais do interior da mata a partir de sua vocalização, que foi gravada (em 14/12/05 e 18/09/06).

Poospiza cinerea (Emberizidae). É considerada globalmente vulnerável (BirdLife International 2008) bem como em Minas Gerais (Machado et al. 1998). Além disso, a espécie apresenta baixa densidade populacional, fator que pode se tornar limitante e significativo para seu processo de extinção (Machado et al. 1998). Conhecida como capacetinho-do-oco-do-pau, essa espécie foi observada apenas uma vez (em 22/04/06) na área de encosta adjacente ao condomínio residencial Quintas do Sol, onde se desenvolve vegetação de Cerrado do tipo campo sujo.

Embernagra longicauda (Emberizidae). Espécie considerada globalmente quase-ameaçada de extinção (BirdLife International 2000, 2008). Essa espécie, popularmente conhecida como rabomole-da-serra, é encontrada freqüentemente acima de $900 \mathrm{~m}$ de altitude, nos campos ferruginosos da MSP, onde suas vocalizações foram gravadas.

Foram registradas nas seis UC's analisadas 25 espécies ameaçadas de extinção (Anexo 2), sendo 13 exclusivas de uma única reserva (cinco do Caraça, seis do PEI, uma do PESRM e outra da MSP); assim, $85 \%$ das espécies ameaçadas exclusivas de uma UC encontram-se somente em uma das reservas grandes. Quarenta por cento das espécies ameaçadas foram registradas exclusivamente nas duas maiores reservas. Três espécies ameaçadas são endêmicas do Cerrado, 13 da Mata Atlântica, e uma dos topos de montanha do sudeste do Brasil. As espécies ameaçadas registradas na MSP representam $16,7 \%$ das ameaçadas registradas nas seis UC's, sendo que C. robustus, possui registro exclusivo nessa reserva, $S$. indigoticus foi registrada também em PEI, EPDA Peti e RPPN Caraça, $P$. cinerea foi registrada também em RPPN Caraça e PPSC, e E. longicauda está presente em todas as UC's aqui analisadas. O número de espécies ameaçadas cresce com a área das reservas $\left(F=21,3522 ; R^{2}=0,8422 ; p=0,0111\right)$, sendo que a variável preditiva (área) explica $82 \%$ da variação do número de espécies ameaçadas (Figura 3).

Juntas, MSP, PEI, RPPN Caraça, EPDA Peti, PPSC e PESRM possuem registro de 81 espécies de aves endêmicas (Anexo 2, Tabela 2). Dezenove espécies endêmicas da Mata Atlântica foram registradas somente em uma das UC's (uma em MSP, três em Peti, oito em PEI, e sete em Caraça); assim, 79\% das espécies endêmicas da Mata Atlântica exclusivas de uma reserva somente nas duas reservas grandes. Três espécies endêmicas do Cerrado foram registradas em apenas uma UC (uma em PESRM e outra no PEI, ambas ameaçadas e uma no Caraça). Quarenta e dois por cento do total de espécies endêmicas foram registradas exclusivamente nas duas maiores reservas. O número de espécies endêmicas da Mata Atlântica nas UC's analisadas cresce com a área da reserva e com a diminuição da longitude $\left(F=14,4060 ; R^{2}=0,9057 ; p=0,0283\right)$, sendo $91 \%$ da variação do número de espécies ameaçadas explicado pelas variáveis preditivas (área e longitude).

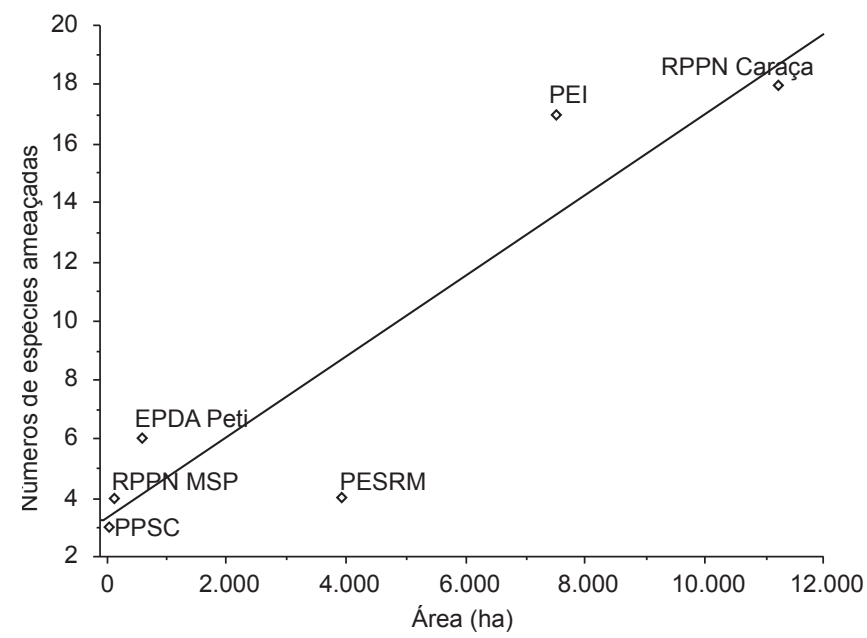

Figura 3. Relação entre número de espécies ameaçadas e área das Unidades de Conservação avaliadas. A regressão linear é significativa $(F=18,6275$; $\left.\mathrm{R}^{2}=0,8232 ; \mathrm{p}=0,0137\right)$.

Figure 3. Relationship between threatened species number and area of the conservation units examined. The linear regression are significant $(\mathrm{F}=18.6275$; $\left.\mathrm{R}^{2}=0.8232 ; \mathrm{p}=0.0137\right)$. 
Ferreira, J.D. et al.

Tabela 2. Número de espécies endêmicas e de ameaçadas registradas em seis Unidades de Conservação do Quadrilátero Ferrífero.

Table 2. Number of endemic and threatened species of six conservation units in Iron Quadrangle region.

\begin{tabular}{lrrrrrrr}
\hline \multicolumn{1}{c}{ Número de espécies } & \multicolumn{9}{c}{ Unidades de conservação } \\
\cline { 2 - 8 } & PESRM & PPSC & MSP & PEI & $\begin{array}{c}\text { RPPN } \\
\text { Caraça }\end{array}$ & EPDA Peti & Total \\
\hline Endêmicas ATL & 18 & 11 & 28 & 57 & 59 & 37 & 71 \\
Endêmicas CE & 5 & 4 & 3 & 2 & 5 & 2 & 8 \\
Endêmicas TM & 2 & 2 & 1 & 2 & 2 & 1 & 2 \\
\hline
\end{tabular}

\section{Discussão}

$\mathrm{O}$ ambiente da MSP que apresentou maior riqueza de aves foi "mata", onde foram registradas com exclusividade 83 espécies, o que indica dependência florestal de grande parte da comunidade de aves presente na reserva. A maior riqueza desse hábitat pode estar relacionada à extensão da área florestal, que ocupa grande parte da MSP. Além disso, as matas possuem maior heterogeneidade espacial, oferecendo uma quantidade maior de micro-hábitats que ambientes mais simples (MacArthur \& MacArthur 1961, MacArthur et al. 1962, August 1983) permitindo o estabelecimento de maior número de espécies (Pianka 2000). Muitas espécies foram encontradas com exclusividade nos outros ambientes amostrados, o que contribui para a diversidade beta da reserva. Os campos ferruginosos apresentaram a menor riqueza de espécies de aves da MSP, o que provavelmente está relacionado às condições extremas enfrentadas nesse ambiente, além da pequena área que ocupa. As condições edafo-climáticas dos campos ferruginosos são severas, ocorrendo alta radiação solar, variação termal diária do substrato que pode alcançar $45^{\circ} \mathrm{C}$, rápida perda de água e solo pobre com alta concentração de metais pesados (Jacobi et al. 2007). Apesar da baixa diversidade de aves, várias das espécies registradas foram observadas exclusivamente nesse ambiente, o que reforça a importância de uma Unidade de Conservação abranger os diversos hábitats de uma região.

O padrão de distribuição das frequiências de ocorrência das aves da MSP, com poucas espécies abundantes e comuns e muitas raras e ocasionais, está de acordo com o encontrado em ambientes tropicais (Janzen 1980, Begon et al. 1996, Macedo 2002).

A ausência sazonal de uma espécie num local ou região é um dos principais indicativos de migração. De fato, a grande maioria das espécies migratórias registradas na MSP não foi registrada em uma ou mais estações. No entanto, a falta de registro de algumas espécies em certas estações pode estar relacionada à raridade ou à baixa atividade geral ou sazonal de algumas aves, e não a movimentos migratórios. Scytalopus indigoticus, por exemplo, foi registrado somente em dois meses do ano na MSP, contudo a ausência de registros nos outros não se deve à migração, mas à baixa atividade e densidade dessa ave. Outro fator que dificulta detecção de atividade migratória é a migração de somente alguns indivíduos da população, já que a espécie continua sendo registrada. Por isso, é importante que sejam realizados estudos de longo prazo, que permitam detectar flutuações anuais e espécies de ocorrência supra-anual (Alves 2007). Muitos registros foram exclusivos de certa estação, revelando que somente um inventário mais completo deve abranger todas elas. Por exemplo, espécies migratórias como $H$. unicolor e $T$. subalaris não seriam registradas se o mês em que apareceram na reserva não fosse amostrado.

Espécies que realizam migração local ou regional na América do Sul possuem poucos representantes ameaçados, com exceção das dependentes da floração do bambu. Isso porque a fragmentação aumenta dramaticamente a distância entre as manchas desse recurso e torna a floração do bambu, fenômeno de ciclo longo (Janzen 1976,
Vasconcelos et al. 2005, Areta et al. 2008), ainda mais rara e imprevisível. Haplospiza unicolor, registrada na MSP, é citada por Stotz et al. (1996) entre as especialistas em sementes de bambu que correm maior risco de extinção. Essa espécie possui registros também em PEI e RPPN Caraça, sendo que na última localidade foi observada grande concentração de indivíduos em época de frutificação da taquara Chusquea attenuata (Vasconcelos et al. 2005). Olmos (1996) relatou a associação de $H$. unicolor a outra espécie do gênero, $C$. meyeriana, na Fazenda Intervales, área de Mata Atlântica de São Paulo, encontrando sincronia na reprodução dessa ave com a do bambu.

As espécies mais frequentemente capturadas no presente estudo foram P. leucoptera, B. hypoleucus, C. lineata e T. melanops. No levantamento de aves realizado na EPDA Peti (altitude média de $800 \mathrm{~m}$ ) a espécie mais capturada também foi $P$. leucoptera, a segunda foi Platyrinchus mystaceus Vieillot, 1818 e a terceira B. hypoleucus (Faria et al. 2006). Em contrapartida, na MSP P. mystaceus foi capturada somente duas vezes. O estudo de Machado \& Fonseca (2000) em fragmentos de Mata Atlântica no vale do rio Doce, localizado a leste do QF revelou que as áreas amostradas de maiores altitudes, que são mais próximas a Peti e a MSP, tiveram maior semelhança em indivíduos capturados. A área denominada "Nova Era" (500 ha, $800 \mathrm{~m}$ ) teve como espécies mais capturadas . leucoptera, seguida de $P$. mystaceus e Chiroxiphia caudata (Shaw \& Nodder, 1793) e a área denominada "Antônio Dias" (300 ha, 750 m), P. leucoptera, seguida de T. melanops e $C$. lineata. As outras áreas amostradas, mais distantes e com menor média altitudinal, Caratinga ( 880 ha e $480 \mathrm{~m}$ ) e Parque Estadual do Rio Doce (35.000 ha e 450 m), não tiveram essas espécies entre as capturadas (a não ser um único individuo de T. melanops capturado na última localidade). Assim, Pyriglena leucoptera foi a mais abundante entre as espécies capturadas nos quatro fragmentos de maiores altitudes. Willis (1979) não observou essa espécie em fragmentos menores que 300 ha na Mata Atlântica paulista e associou esse fato a ausência de formigas de correição em áreas pequenas. Por outro lado, $P$. leucoptera foi registrada em abundância na MSP, apesar da reserva possuir somente 147 ha e de não terem sido observadas ali formigas de correição.

Foi encontrada baixa representatividade de grande número de espécies ameaçadas nas áreas protegidas. A presença de uma espécie em muitos fragmentos reduz sua probabilidade de extinção, por aumentar o número de populações da mesma (Primack \& Rodrigues 2001). O resultado expõe a importância de um sistema de reservas planejado de modo a abranger toda a diversidade geográfica e ecológica da região (Jennings 2000, Scott et al. 2001). O conhecimento da composição regional da biota é essencial para tal planejamento, que almeja preencher as lacunas na proteção da biodiversidade, criando novas áreas de proteção ambiental que incluam espécies mal representadas nas UC's existentes.

O parâmetro área possui reconhecida importância na persistência das espécies de aves em ambientes fragmentados (Karr 1982, Stratford \& Stouffer 1999, Uezu et al. 2005), cada vez mais freqüentes com o avanço da modificação da terra para uso humano. O presente estudo 
mostra que o número de espécies ameaçadas é maior quanto maior a área das reservas analisadas, efeito esperado pela relação espécie-área (MacArthur \& Wilson 1963). Encontrou-se ainda que grande parte das espécies ameaçadas foi registrada somente nas maiores reservas. Esse resultado reforça que pequenas UC's são incapazes de proteger toda a biodiversidade da região, sugerindo que a proteção de grandes áreas é indispensável para a conservação efetiva das espécies e que deve ser, portanto, priorizada.

O número de espécies ameaçadas do PESRM demonstrou o maior valor de resíduo da regressão linear simples, apresentando menos espécies do que o esperado de acordo com seu tamanho. Isso pode ser reflexo do pequeno esforço de amostragem do levantamento, realizado em uma avaliação ecológica rápida por nove dias consecutivos, para o plano de manejo do parque (Hass et al. 2005). Existem acentuadas diferenças entre os esforços amostrais dos inventários, que certamente influenciam os resultados e devem ser rememoradas em suas análises. Inventários com amostragens pequenas freqüentemente possuem curvas de acumulação de espécies que não apresentam assíntota óbvia, indicando que várias espécies não foram detectadas. Isso dificulta a comparação da riqueza de espécies a outros locais, sendo aconselhável o uso de estimadores para reduzir o efeito do tamanho da amostragem (Colwell \& Coddigton 1994).

A ocorrência de espécies ameaçadas nas UC's citadas indica que essas reservas fazem parte de suas áreas de vida, sendo úteis para os esforços de conservação das mesmas. São necessários, no entanto, estudos demográficos das espécies para avaliar se as populações serão capazes de persistir no local (Brooks et al. 1999). A MSP, por exemplo, é uma área fragmentada e pequena, e as populações podem não ter um tamanho mínimo viável, o que as torna mais sujeitas à extinção pelos efeitos da perda de variabilidade genética, variações demográficas e aleatoriedade ambiental (Pimm et al. 1988). Porém, próximo à MSP existe uma porção maior de mata protegida (RPPN Mata do Jambreiro, 902 ha) que, apesar de também ser uma área fragmentada, provavelmente contribui para o fluxo gênico de algumas espécies, reduzindo os efeitos da fragmentação. Por outro lado, durante o estudo, uma área não protegida adjacente a MSP começou a sofrer um processo de urbanização, sendo transformada em condomínio residencial. Parte do terreno desmatado era composta de ambiente florestal contíguo à mata em estudo e se localiza entre a MSP e a Mata do Jambreiro, o que aumenta o isolamento das reservas.

As seis UC's analisadas apresentam espécies endêmicas da Mata Atlântica e do Cerrado, reflexo do caráter ecotonal da região. As espécies endêmicas estão mais gravemente sujeitas à extinção do que as demais, já que ocorre extinção global quando a espécie desaparece do bioma ao qual é restrita (Pimm \& Askins 1995). Esses registros sugerem a importância dessas UC's na preservação de espécies endêmicas de dois biomas brasileiros prioritários para conservação.

A taxa de endemismo da Mata Atlântica é um os maiores do planeta, enquanto que no Cerrado existe número relativamente baixo de espécies endêmicas (Myers et al. 2000). Isso é refletido na composição de espécies endêmicas das UC's analisadas, onde o número de espécies restritas à Mata Atlântica é superior ao do Cerrado. A riqueza de espécies endêmicas da Mata Atlântica é influenciada pelo tamanho da UC, assim como a riqueza de espécies ameaçadas, mas, adicionalmente, depende da longitude em que a reserva está localizada. Assim, o PESRM e o PPSC possuem poucas espécies endêmicas da Mata Atlântica se comparados às UC's localizadas mais a leste. Por outro lado, juntos apresentam seis das oito espécies endêmicas do Cerrado e as duas espécies endêmicas dos topos de montanha do sudeste do Brasil. EPDA Peti, PEI e RPPN Caraça, localizadas mais a leste, apresentam mais espécies endêmicas da Mata Atlântica, sendo que as duas últimas (áreas maiores) possuem os maiores números de endemismo entre as seis UC's.
Além da diferença amostral das UC's é importante ressaltar que suas áreas consideradas nas análises constituem limites artificiais e que não foi calculada a extensão dos tipos de vegetação das reservas, o que certamente influencia na composição da avifauna. A área é, portanto, uma medida inexata, mas não deixa de refletir a grandeza aproximada das reservas e de suas biodiversidades.

$\mathrm{O}$ presente estudo enriquece o conhecimento da avifauna do Estado de Minas Gerais, tanto do domínio do Cerrado quanto da Mata Atlântica, especificamente do QF. Esta região sofre uma grande pressão econômica e está sendo rapidamente transformada pela urbanização e mineração (Jacobi \& Carmo 2008). Os campos ferruginosos são especialmente ameaçados, pois são associados a solos de elevada qualidade mineral e constituem um ambiente bastante localizado. São encontrados em Minas Gerais principalmente no Quadrilátero Ferrífero e, em menores quantidades, ao longo do Espinhaço meridional nas regiões de Serro e Capelinha. No resto do Brasil estão em Carajás, no Pará, e em Corumbá, no Mato Grosso do Sul (Castro 2008). A MSP protege somente uma pequena área de campos ferruginosos, e são poucas as Unidades de Conservação que os incluem. Cerca de $90 \%$ das áreas que possuem campos ferruginosos têm direitos de mineração concedidos a indústrias e a demanda de minério só tende a crescer (DNPM 2006, Jacobi \& Carmo 2008). Além desse ambiente peculiar existem no QF áreas consideráveis de Cerrado e de Mata Atlântica, reconhecidas como prioritárias à conservação. As acentuadas pressões econômicas e demográficas exercidas na região tornam urgentes as ações para preservar a região do QF. $\mathrm{O}$ levantamento efetivo de espécies, abrangendo todas as fitofisionomias e estações do ano, é uma das medidas conservacionistas sugeridas, pois fornece base para um planejamento eficiente de novas UC's.

\section{Agradecimentos}

Agradecemos à AngloGold Ashanti e à R. Guadalupe por viabilizarem o estudo; à C. M. A. Faria e M. F. Vasconcelos pela ajuda na identificação de algumas espécies; aos estagiários voluntários (F. Alves, E. Mesquita, M. Butti, P. Rodrigues, I. Mortimer, I. Vasconcelos) pela grande ajuda em campo; aos funcionários da MSP; ao Centro de Pesquisa para Conservação de Aves Silvestres (CEMAVE-IBAMA) pela concessão de anilhas e licença para anilhamento de aves silvestres; ao laboratório de Geoprocessamento da UFMG, onde foi desenvolvido o mapa da área de estudo. Agradecemos ainda à M.F. Vasconcelos pelas sugestões, comentários e revisão do texto e J. A. Mobley pela revisão do abstract. J. D. F. agradece ao CNPq pela bolsa de mestrado. M.R. agradece ao CNPq (processo n. 473428/2004-0 e 300731/2006-0) a Fapemig (PPM CRA APQ-0434-5.03/07) e a Fundação O Boticário de Proteção à Natureza pelo apoio ao Laboratório de Ornitologia da UFMG.

\section{Referências Bibliográficas}

ALVES, M.A.S. 2007. Sistemas de migrações de aves em ambientes terrestres no Brasil: exemplos, lacunas e propostas para o avanço do conhecimento. Ararajuba: revista brasileira de ornitologia 15(2):231-238.

ARETA, J.I., BODRATI, A. \& COCKLE, K. 2008. Specialization on Guadua bamboo seeds by three bird species in the Atlantic Forest of Argentina. Biotropica 41(1):66-73.

AUGUST, P.V. 1983. The role of habitat complexity and heterogeneity in structuring tropical mammal communities. Ecology 64(6):1495-1507.

AYRES, M., AYRES, D.L. \& SANTOS, A.A.S. 2007. BioEstat: aplicações estatísticas na área de ciências bio-médicas. Belém.

BEGON, M., HARPER, J.L. \& TOWNSEND, C.R. 1996. Ecology: individuals, populations and communities. Blackwell, Oxford, p.945.

Birdlife International. 2000. Threatened birds of the world. Lynx Editions, Cambridge, p.852. 
Birdlife International. 2008. BirdLife's online World Bird Database: the site for bird conservation. Version 2.1. Cambridge. http://www.birdlife.org. (último acesso em 20/05/2008).

BROOKS, T., PIMM, S.L. \& OYUGI, J.O. 1999. Time lag between deforestation and bird extinction in tropical forest fragments. Conserv. Biol. 13(5):1140-1150.

BURTON, R. 1869. Explorations of the highlands of Brazil with a full account of the gold and diamond mines. Also canoeing down 1.500 miles of the great São Francisco river from Sabará to the sea. Tinsley Brothers, London, p.443. (v. 2).

CARNEVALLI, N.E.D. 1980. Contribuição ao estudo da ornitofauna da Serra do Caraça, Minas Gerais. Lundiana 1(1):89-98.

CARNEVALLI, N.E.D., MACHADO, R.B., BRANDT, A., LAMAS, I.R., LINS, L.V., BARROS, L.P. \& SOUZA, A.L.T. 1989. Estudo qualitativo da avifauna da Estação de Pesquisa e Desenvolvimento Ambiental de Peti. UFMG/CEMIG CEMIG, Belo Horizonte, p.65.

CASTRO, P.T.A. 2008. Cangas: a influência da geodiversidade na biodiversidade. In I Simpósio Aforamentos ferruginosos no Quadrilátero Ferrífero: biodiversidade, conservação e perspectivas de sustentabilidade. Universidade Federal de Minas Gerais, Belo Horizonte. (CD-ROM).

CHESSER, R.T. 1994. Migration in South America: an overview of the austral system. Bird conserv. int. 4:91-107.

COLWELL, R.K. 2006. Estimates: statistical estimation of species richness and shared species from samples. Version 8.0. http://viceroy.eeb.uconn. edu/estimates (último acesso em 20/05/2008).

COLWELL, R.K \& CODDINGTON, J.A. 1994. Estimating terrestrial biodiversity through extrapolation. Philosophical transactions of the Royal Society of London. Series B - Biological sciences 355(1311):101-118.

Comitê Brasileiro de Registros Ornitológicos - CBRO. 2008. Listas das aves do Brasil. http://www.cbro.org.br. (último acesso em 23/01/2009).

DEAN, W. 2002. A ferro e fogo: a história e a devastação da Mata Atlântica brasileira. Companhia das Letras, São Paulo, p.484.

Departamento Nacional de Produção Mineral - DNPM. 2006. Anuário mineral brasileiro. http://www.dnpm.gov.br/conteudo. asp?IDSecao=68\&IDPagina=789. (último acesso em 01/02/2009).

DRUMMOND, G.M., MARTINS, C.S., MACHADO, A.B.M., SEBAIO, F.A. \& ANTONINI, Y. 2005. Biodiversidade em Minas Gerais: um atlas para sua conservação. Fundação Biodiversitas, Belo Horizonte, p.222.

FARIA, C.M.A., RODRIGUES, M., AMARAL, F.Q., MÓDENA, E. \& FERNANDES, A.M. 2006. Aves de um fragmento de Mata Atlântica no alto Rio Doce, Minas Gerais: colonização e extinção. Rev. Bras. Zool. 23(4):1217-1230.

FIGUEREDO, J.B. \& SALINO, A. 2005. Pteridófitas de quatro reservas particulares do patrimônio natural ao sul da região metropolitana de Belo Horizonte, Minas Gerais, Brasil. Lundiana 6(2):83-94.

GONZAGA, L.P. \& CASTIGLIONI, G. 2001. Aves das montanhas do sudeste do Brasil. Universidade Federal do Rio de Janeiro, Rio de Janeiro. (CD-ROM).

HASS, A., FARIA, C., FARIA, L.P., HOFFMAN, D. \& GOMES, H.B. 2005. Avaliação ecológica rápida para o plano de manejo do Parque Estadual da Serra do Rola-Moça: relatório do grupo temático aves. In Plano de Manejo do Parque Estadual da Serra do Rola - Moça. (Relatório não publicado).

HELTSHE, J.F. \& FORRESTER, N.E. 1983. Estimating specie richness using the Jakknife procedure. Biometrics 39(1):1-11.

JACOBI, C.M. \& CARMO, F.F. 2008. The contribution of ironstone outcrops to plant diversity in the Iron Quadrangle, a Threatened Brazilian Landscape. Ambio 37(4):324-326.

JACOBI, C.M., CARMO, F.F., VINCENT, R.C. \& STEHMANN, J.R. 2007. Plant communities on ironstone outcrops: a diverse and endangered Brazilian ecosystem. Biodiversity and Conservation 16(7):2185-2200.

JANZEN, D.H. 1976. Why bamboos wait so long to flower? Ann. Rev. Ecolog. Syst. 7:347-391.

JANZEN, D.H. 1980. Ecologia vegetal nos trópicos. Universidade de São Paulo, São Paulo, p.93.
JENNINGS, M.D. 2000. Gap analysis: concepts, methods, and recent results. Landsc. ecol. 15(1):5-20.

KARR, J.R. 1982. Avian extinction on Barro Colorado Island, Panama: a reassessment. The American Naturalist 119(2):220-239.

LOPES, L.E. 2008. The range of the curl-crested jay: lessons for evaluating bird endemism in the South American Cerrado. Divers. Distrib. 14(4):561-568.

MACARTHUR, R.H. \& MACARTHUR, J.W. 1961. On bird species diversity. Ecology 42(3):594-598.

MACARTHUR, R.H., MACARTHUR, J.W. \& PREER, J. 1962. On bird species diversity: II predictions of bird census from habitat measurements. The American Naturalist 96(888):167-174.

MACARTHUR, R.H. \& WILSON, E.O. 1963. An equilibrium theory of insular zoogeography. Evolution 17(4):373-387.

MACEDO, R.H.F. 2002. The avifauna: ecology, biogeography, and behavior. In The cerrados of brazil: ecology and natural history of a neotropical savanna (P.S. Oliveira \& R.J. Marquis, eds.). Columbia University Press, Nova York, p. 242-265.

MACHADO, A.B.M., FONSECA, G.A.B., MACHADO, R.B., AGUIAR, L.M.S. \& LINS, L.V. 1998. Livro vermelho das espécies ameaçadas em Minas Gerais. Fundação Biodiversitas, Belo Horizonte, p.605.

MACHADO, R.B. \& FONSECA, G.A.B. 2000. The avifauna of Rio Doce Valley, southeastern Brazil, a highly fragmented area. Biotropica 32(4b):914-924.

MARINI, M.A. \& GARCIA, F.I. 2005. Bird Conservation in Brazil. Conserv. Biol. 19(3):665-671.

MYERS, N., MITTERMEIER, R.A., MITTERMEIER, C.G., FONSECA, G.A.B. \& KENT, J. 2000. Biodiversity hotspots for conservation priorities. Nature 403(6772):853-858.

OLMOS, F. 1996. Satiation or deception? Mast-seeding Chusquea bamboos, birds and rats in the Atlantic forest. Rev. bras. biol. 56(2):391-401.

PIANKA, E.R. 2000. Evolutionary ecology. 6 ed. Adison Wesley Educational Publishers, San Francisco, p.212.

PIMM, S.L. \& ASKINS, R.A. 1995. Forest losses predict bird extinction in eastern North America. PNAS 92(20):9343-9347.

PIMM, S.L., JONES, H.L. \& DIAMOND, J. 1988. On the risk of extinction. The American Naturalist 132(6):757-785.

PRIMACK, R.B. \& RODRIGUES, E. 2001. Biologia da conservação. Midiograf, Londrina, p.327.

RIBON, R. 2006. Relatório Avifauna. In Plano de manejo do Parque Estadual do Itacolomi. Fundação Biodiversitas /UFOP/IEF, Belo Horizonte, p.42.

RODRIGUES, M., CARRARA, L.A., FARIA, L.P. \& GOMES, H.B. 2005. As aves do Parque Nacional da Serra do Cipó: o vale do Rio Cipó, Minas Gerais, Brasil. Rev. Bras. Zool. 22(2):326-338.

RODRIGUES, M. \& MICHELIN, V. 2005. Riqueza e diversidade de aves aquáticas de uma lagoa natural no sudeste do Brasil. Rev. Bras. Zool. 22(4):928-935.

SCOTT, J.M., DAVIS, F.W., MCGHIE, R.G., WRIGHT, R.G., GROVES, C. \& ESTES, J. 2001. Nature reserves: do they capture the full range of America's biological diversity? Ecol. appl. 11(4):999-1007.

SILVA, J.M.C. \& BATES, J.M. 2002. Biogeographic patterns and conservation in the South American Cerrado: a tropical Savanna hotspot. BioScience 52(3):225-233.

SMITH, E.P. \& Van BELLE, G. 1984. Nonparametric estimation of species richness. Biometrics 40(1):119-129.

SOKAL, R.R. \& ROHLF, F.J. 1995. Biometry. 3 ed. Freeman \& Co, Nova York, p.887.

SOUZA, R.G.V. \& BRITO, F.R.A. 2006. A expansão urbana da região metropolitana de Belo Horizonte e suas implicações para a redistribuição para a redistribuição espacial da população: a migração dos ricos. In XV Encontro de Estudos Populacionais. ABEP, Caxambu, p.117. 
STOTZ, D.F., FITZPATRICK, J.W., PARKER III, T.A. \& MOSKOVITS, D.K. 1996. Neotropical Birds: ecology and conservation. The University of Chicago Press, Chicago, p.882.

STRATFORD, J.A. \& STOUFFER, P.C. 1999. Local extinctions of terrestrial insectivorous birds in a fragmented landscape near Manaus, Brazil. Conserv. Biol. 13(6): 1416-1423.

UEZU, A., METZGER, J.P. \& VIELLIARD, J.M.E. 2005. Effects of structura and functional connectivity and patch size on the abundance of seven Atlantic Forest bird species. Biol. Conserv. 123(4):507-519.

VASCONCELOS, D. 1999. História antiga das Minas Gerais. Itatiaia, Belo Horizonte, p. 283.

VASCONCELOS, M.F. \& MELO Jr., T.A. 2001. An ornithological survey of Serra do Caraça, Minas Gerais, Brazil. Cotinga 15(1):21-31.

VASCONCELOS, M.F., MALDONADO-COELHO, M. \& BUZZETTI, D.C.R. 2003a. Range extensions for the gray-blacked tachuri (Polystictus superciliaris) and the pale-throated-serra-finch (Embernagra longicauda) with a revision on their geographic distribution. Ornitol. Neotrop. 14(3):477-489.

VASCONCELOS, M.F, VASCONCELOS, P.N., MAURÍCIO, G.M., MATRANGOLO, C.A.R., DELL'AMORE, C.M., NEMÉSIO, A., FERREIRA, J.C. \& ENDRIGO, E. 2003b. Novos registros ornitológicos para a Serra do Caraça, Brasil, com comentários sobre distribuição geográfica de algumas espécies. Lundiana 4(2):135-139.

VASCONCELOS, M.F., VASCONCELOS, A.P., VIANA, P.L., PALÚ, L. \& SILVA, J.F. 2005. Observações sobre aves granívoras (Columbidae e Emberizidae) associadas à frutificação de taquaras (Poaceae,
Bambusoideae) na porção meridional da Cadeia do Espinhaço, Minas Gerais, Brasil. Lundiana 6(1):75-77.

VASCONCELOS, M.F. 2007a. Aves observadas no Parque Paredão da Serra do Curral, Belo Horizonte, Minas Gerais, Brasil. Atual. ornitol. 136(1):6-11.

VASCONCELOS, M.F. 2007b. Comentários sobre a avifauna da Estação de Pesquisa e Desenvolvimento Ambiental de Peti, Minas Gerais, com a lista dos exemplares coletados na região. Atual. Ornitol. 137(1):7-9.

VIELLIARD, J.M. 1995. Guia sonoro das aves do Brasil. Sociedade Brasileira de Ornitologia e Fundação O Boticário de Proteção à Natureza, Campinas. (CD-ROM 1).

VIELLIARD, J.M. 2002. Vozes das aves do Brasil. Universidade de Campinas e Academia Brasileira de Ciências, Campinas. (CD-ROM).

XENO-CANTO. 2008. http://www.xeno-canto.org (último acesso em 20/05/2008).

WHITNEY, B.M., PACHECO, J.F. \& PARRINI, R. 1995. Two species of Neopelma in southeastern Brazil and diversification within the Neopelma/ Tyranneutes complex: implications of the subspecies concepts for conservation (Passeriformes: Tyrannidae). Ararajuba: revista brasileira de ornitologia 3(1):43-53.

WILLIS, E.O. 1979. The composition of avian communities in remanescent woodlots in southern Brazil. Pap. avulsos zool. 27(7):1-25.

Recebido em 05/09/08

Versão reformulada recebida em 15/06/09

Publicado em 01/07/09 
Anexo 1. Lista de espécies de aves registradas na RPPN Mata Samuel de Paula (MG), situações de endemismo e conservação, ambientes(s), meio(s) de registro, situação migratória e frequiência de ocorrência. Conservação: (gq) quase-ameaçado, (gp) em perigo e (gv) vulnerável à extinção no mundo (BirdLife International 2008); (rp) em perigo e (rv) vulnerável à extinção em Minas Gerais (Machado et al. 1998). Endemismo: (ATL) endêmica da Mata Atlântica, segundo Stotz et al. (1996); (TM) endêmica dos topos de montanha do Sudeste do Brasil e (CE) endêmica do Cerrado, segundo Silva (2002) e Vasconcelos et al. (2003a). Ambiente de registro: (D) área aberta degradada, (M) mata, (C) cerrado, (F) campos ferruginosos. Registro: (G) gravado, (F) fotografado. Situação migratória: (a) migrante austral, segundo Chesser (1994); (n) migrante neártica, (np) migrante neártica parcial e (mr) migrante regional, segundo Stotz et al. (1996). Freqüência: (A) abundante, (C) comum, (E) escassa, (R) rara e (O) ocasional. Classificação taxonômica segundo CBRO (2008).

Annex 1. Birds species recorded at RPPN Mata Samuel de Paula, Minas Gerais state, Brazil, including their endemism and conservation status, habitat(s), record method(s), migratory status, and frequency of occurrence. Conservation: globally (gq) near-threatened, (gp) endangered, and (gv) vulnerable (BirdLife International 2008); regionally (at Minas Gerais state) (rp) endangered, and (rv) vulnerable (Machado et al. 1998). Endemism: (ATL) Atlantic Forest endemics, according to Stotz et al. (1996); (TM) top mountain of southeastern Brazil endemics, and (CE) Cerrado endemics, according to Silva (2002) and Vasconcelos et al. (2003a). Habitat records: (D) degraded open vegetation, (M) forest, (C) savanna, (F) ferrugineous rocky fields. Records: (G) vocalization recorded, (F) photographed. Migratory status: (a) austral migrant, according to Chesser (1994); (n) neartic migrant, (np) partial neartic migrant, and (mr) regional migrant, according to Stotz et al. (1996). Frequency: (A) abundant, (C) common, (E) uncommon, (R) rare, and (O) occasional. Taxonomic classification according to CBRO (2008).

Famílias e espécies $\quad$ Nome popular $\quad$ Endemismo/ Ambiente Registro Migração Freqüência

Tinamidae Gray, 1840 (23) Conservação

Crypturellus obsoletus (Temminck, 1815)

Cracidae Rafinesque, 1815

Penelope sp.

Cathartidae Lafresnaye, 1839

Cathartes aura (Linnaeus, 1758)

Coragyps atratus (Bechstein, 1793)

Accipitridae Vigors, 1824

Rupornis magnirostris (Gmelin, 1788)

Buteo albicaudatus Vieillot, 1816

Buteo albonotatus Kaup, 1847

Falconidae Leach, 1820

Caracara plancus (Miller, 1777)

Milvago chimachima (Vieillot, 1816)

Herpetotheres cachinnans

(Linnaeus, 1758)

Micrastur semitorquatus (Vieillot, 1817)

Falco sp.

Rallidae Rafinesque, 1815

Aramides saracura (Spix, 1825)

Cariamidae Bonaparte, 1850

Cariama cristata (Linnaeus, 1766)

Charadriidae Leach, 1820

Vanellus chilensis (Molina, 1782)

Columbidae Leach, 1820 (23)

Columbina talpacoti (Temminck, 1811)

Columbina squammata (Lesson, 1831)

Patagioenas picazuro (Temminck, 1813)

Patagioenas plumbea (Vieillot, 1818)

Leptotila verreauxi Bonaparte, 1855

Leptotila rufaxilla

(Richard \& Bernard, 1792)

Psittacidae Rafinesque, 1815

Aratinga leucophthalma

(Statius Muller, 1776)

Forpus xanthopterygius (Spix, 1824)

Brotogeris chiriri (Vieillot, 1818) inhambuguaçu

urubu-de-cabeça-vermelha
urubu-de-cabeça-preta
gavião-carijó
gavião-de-rabo-branco
gavião-de-rabo-barrado

caracara
carrapateiro
Acauã

falcão-relógio

saracura-do-mato

seriema

quero-quero

rolinha-roxa

fogo-apagou

pombão

pomba-amargosa

juriti-pupu

juriti-gemedeira

periquitão-maracanã

tuim

periquito-de-encontroamarelo

maitaca-verde

M

M

M

$$
\text { G }
$$

$\mathrm{R}$

C/D

$\mathrm{M} / \mathrm{C} / \mathrm{D}$

$\mathrm{a} / \mathrm{np}$

R

E

$\begin{array}{cccc}\mathrm{M} / \mathrm{C} & \mathrm{G} & \mathrm{R} \\ \mathrm{F} & & \mathrm{a} & \mathrm{R} \\ \mathrm{F} & & & \mathrm{O}\end{array}$

C

C

C

G

R

E

$\mathrm{R}$

C

G

R

$\mathrm{O}$

ATL

M

G

R

C

G

$\mathrm{R}$

D

a

$\mathrm{R}$

D E

C R

$\begin{array}{lll}\text { C } & \text { G }\end{array}$

M G

M G

M

F

$\mathrm{R}$

Pionus maximiliani (Kuhl, 1820)
M

C

C

M

\section{E}

$\mathrm{R}$

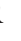

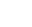

O

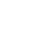

R

(n)

R




\section{Famílias e espécies}

Endemismo/ Ambiente Registro Migração Freqüiência Conservação

Cuculidae Leach, 1820

Piaya cayana (Linnaeus, 1766)

Crotophaga ani Linnaeus, 1758

Tapera naevia (Linnaeus, 1766)

Strigidae Leach, 1820

Megascops choliba (Vieillot, 1817)

Caprimulgidae Vigors, 1825

Nyctidromus albicollis (Gmelin, 1789)

Caprimulgus longirostris Bonaparte, 1825

Apodidae Olphe-Galliard, 1887

Chaetura meridionalis Hellmayr, 1907

Streptoprocne zonaris (Shaw, 1796)

Trochilidae Vigors, 1825 (82)

Phaethornis ruber (Linnaeus, 1758)

Phaethornis pretrei

(Lesson \& Delattre, 1839)

Eupetomena macroura (Gmelin, 1788)

Florisuga fusca (Vieillot, 1817)

Colibri serrirostris (Vieillot, 1816)

Chlorostilbon lucidus (Shaw, 1812)

Thalurania glaucopis (Gmelin, 1788)

Amazilia versicolor (Vieillot, 1818)

Amazilia lactea (Lesson, 1832)

Trogonidae Lesson, 1828

Trogon surrucura Vieillot, 1817

Ramphastidae Vigors, 1825

Ramphastos toco Statius Muller, 1776

Picidae Leach, 1820

Picumnus cirratus Temminck, 1825

Colaptes campestris (Vieillot, 1818)

Campephilus robustus

(Lichtenstein, 1818)

Melanopareiidae Irestedt, Fjeldså, Johansson \& Ericson, 2002

Melanopareia torquata (Wied, 1831)

Thamnophilidae Swainson, 1824 (163)

Mackenziaena leachii (Such, 1825)

Thamnophilus caerulescens Vieillot, 1816

Dysithamnus mentalis (Temminck, 1823)

Herpsilochmus atricapillus Pelzeln, 1868

Formicivora serrana Hellmayr, 1929

Drymophila ferruginea (Temminck, 1822)

Pyriglena leucoptera (Vieillot, 1818)

Conopophagidae Sclater \& Salvin, 1873

Conopophaga lineata (Wied, 1831)

Rhinocryptidae Wetmore, 1930

Scytalopus indigoticus (Wied, 1831)

Dendrocolaptidae Gray, 1840

Sittasomus griseicapillus (Vieillot, 1818)

$$
\begin{gathered}
\text { alma-de-gato } \\
\text { anu-preto } \\
\text { saci }
\end{gathered}
$$

corujinha-do-mato

bacurau

bacurau-da-telha

andorinhão-do-temporal

taperuçu-de-coleira-branca

rabo-branco-rubro

rabo-branco-acanelado

beija-flor-tesoura

beija-flor-preto

beija-flor-de-orelha-violeta

besourinho-de-bicovemelho

beija-flor-de-fronte-violeta

beija-flor-de-banda-branca

beija-flor-de-peito-azul

surucuá-variado

tucanuçu

pica-pau-anão-barrado

pica-pau-do-campo

pica-pau-rei

tapaculo-de-colarinho

borralhara-assobiadora

choca-da-mata

choquinha-lisa

chorozinho-de-

chapéu-preto

formigueiro-da-serra trovoada

papa-taoca-do-sul

chupa-dente

macuquinho

arapaçu-verde
$\mathrm{M}$

$\mathrm{C}$

C

G

E

O

$\mathrm{O}$

M

$\mathrm{O}$

C

C

G/F

E

R

F

M

G

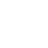

G

$\mathrm{O}$

$\mathrm{O}$

M G/F

E

M G/F

C

ATL

D

F

$\mathrm{O}$

$\mathrm{M}$

$\mathrm{C}$

C/D

G

F

a

R

E

R

ATL

M

F

F

R

R

E

ATL

M

G

R

M

O

M

M

ATL, rp

C

G

A

$\mathrm{O}$

O

CE

F

G

E

ATL

M

$\mathrm{G} / \mathrm{F}$

E

M G/F

A

M

$\mathrm{G}$

E

M

G/F

A

ATL

M

G/F

R

ATL

M

$\mathrm{G}$

R

ATL

M

G/F

A

ATL

M

$\mathrm{G} / \mathrm{F}$

E

ATL, gq

M

G

$\mathrm{R}$

M
$\mathrm{G} / \mathrm{F}$ 


\begin{tabular}{|c|c|c|c|c|c|c|}
\hline Famílias e espécies & Nome popular & $\begin{array}{l}\text { Endemismo/ } \\
\text { Conservação }\end{array}$ & Ambiente & Registro & Migração & Freqüência \\
\hline Xiphocolaptes albicollis (Vieillot, 1818) & arapaçu-de-garganta-branca & & M & G & & $\mathrm{R}$ \\
\hline Xiphorhynchus fuscus (Vieillot, 1818) & arapaçu-rajado & ATL & M & $\mathrm{G}$ & & $\mathrm{R}$ \\
\hline \multicolumn{7}{|l|}{ Furnariidae Gray, 1840 (102) } \\
\hline Furnarius rufus (Gmelin, 1788) & joão-de-barro & & $\mathrm{D}$ & & & $\mathrm{R}$ \\
\hline Synallaxis ruficapilla Vieillot, 1819 & pichororé & ATL & M & $\mathrm{G} / \mathrm{F}$ & & $\mathrm{C}$ \\
\hline Synallaxis cinerascens Temminck, 1823 & pi-puí & & M & G & & $\mathrm{R}$ \\
\hline Synallaxis frontalis Pelzeln, 1859 & petrim & & M & G & a & $\mathrm{R}$ \\
\hline Synallaxis spixi Sclater, 1856 & joão-teneném & & $\mathrm{D}$ & G & & A \\
\hline Phacellodomus rufifrons (Wied, 1821) & joão-de-pau & & $\mathrm{C}$ & G & & $\mathrm{R}$ \\
\hline $\begin{array}{l}\text { Syndactyla rufosuperciliata } \\
\text { (Lafresnaye, 1832) }\end{array}$ & trepador-quiete & & M & $\mathrm{G} / \mathrm{F}$ & & $\mathrm{R}$ \\
\hline Philydor rufum (Vieillot, 1818) & limpa-folha-de-testa-baia & & M & $\mathrm{G} / \mathrm{F}$ & & $\mathrm{R}$ \\
\hline Automolus leucophthalmus (Wied, 1821) & $\begin{array}{l}\text { barranqueiro-de-olho- } \\
\text { branco }\end{array}$ & ATL & M & G & & $\mathrm{E}$ \\
\hline Lochmias nematura (Lichtenstein, 1823) & joão-porca & & M & $\mathrm{G} / \mathrm{F}$ & & A \\
\hline Xenops rutilans Temminck, 1821 & bico-virado-carijó & & M & $\mathrm{G} / \mathrm{F}$ & & $\mathrm{R}$ \\
\hline \multicolumn{7}{|l|}{ Tyrannidae Vigors, 1825} \\
\hline Mionectes rufiventris Cabanis, 1846 & abre-asa-de-cabeça-cinza & ATL & M & G & & $\mathrm{E}$ \\
\hline $\begin{array}{l}\text { Leptopogon amaurocephalus } \\
\text { Tschudi, } 1846\end{array}$ & cabeçudo & & M & $\mathrm{G} / \mathrm{F}$ & & $\mathrm{E}$ \\
\hline Corythopis delalandi (Lesson, 1830) & estalador & & M & $\mathrm{G}$ & & $\mathrm{R}$ \\
\hline Hemitriccus nidipendulus (Wied, 1831) & tachuri-campainha & ATL & M & $\mathrm{F}$ & & $\mathrm{R}$ \\
\hline Myiornis auricularis (Vieillot, 1818) & miudinho & ATL & M & $\mathrm{G} / \mathrm{F}$ & & $\mathrm{E}$ \\
\hline $\begin{array}{l}\text { Poecilotriccus plumbeiceps } \\
\text { (Lafresnaye, 1846) }\end{array}$ & tororó & & M & $\mathrm{G} / \mathrm{F}$ & & A \\
\hline Todirostrum poliocephalum (Wied, 1831) & teque-teque & ATL & M & $\mathrm{G} / \mathrm{F}$ & & $\mathrm{E}$ \\
\hline Phyllomyias fasciatus (Thunberg, 1822) & piolhinho & & M & G & a & $\mathrm{E}$ \\
\hline Myiopagis caniceps (Swainson, 1835) & guaracava-cinzenta & & M & & a & $\mathrm{R}$ \\
\hline Myiopagis viridicata (Vieillot, 1817) & $\begin{array}{l}\text { guaracava-de-crista-alar- } \\
\text { anjada }\end{array}$ & & M & G & a & $\mathrm{O}$ \\
\hline Elaenia flavogaster (Thunberg, 1822) & $\begin{array}{c}\text { guaracava-de-barriga- } \\
\text { amarela }\end{array}$ & & $\mathrm{D}$ & G & a & $\mathrm{C}$ \\
\hline Elaenia cristata Pelzeln, 1868 & $\begin{array}{l}\text { guaracava-de-topete- } \\
\text { uniforme }\end{array}$ & & $\mathrm{C} / \mathrm{D}$ & G & & $\mathrm{R}$ \\
\hline $\begin{array}{l}\text { Elaenia obscura } \\
\text { (d'Orbigny \& Lafresnaye, 1837) }\end{array}$ & tucão & & $\mathrm{M} / \mathrm{C}$ & $\mathrm{G} / \mathrm{F}$ & & $\mathrm{R}$ \\
\hline $\begin{array}{l}\text { Camptostoma obsoletum } \\
\text { (Temminck, 1824) }\end{array}$ & risadinha & & $\mathrm{M} / \mathrm{C} / \mathrm{D}$ & G & a & $\mathrm{C}$ \\
\hline Serpophaga subcristata (Vieillot, 1817) & alegrinho & & M & $\mathrm{G} / \mathrm{F}$ & a & $\mathrm{R}$ \\
\hline Phaeomyias murina (Spix, 1825) & bagageiro & & M & $\mathrm{F}$ & a & $\mathrm{O}$ \\
\hline Phylloscartes ventralis (Temminck, 1824) & borboletinha-do-mato & & M & G & & $\mathrm{R}$ \\
\hline Tolmomyias sulphurescens (Spix, 1825) & bico-chato-de-orelha-preta & & M & $\mathrm{G} / \mathrm{F}$ & & A \\
\hline Platyrinchus mystaceus Vieillot, 1818 & patinho & & M & $\mathrm{G} / \mathrm{F}$ & & $\mathrm{C}$ \\
\hline $\begin{array}{l}\text { Myiophobus fasciatus } \\
\text { (Statius Muller, 1776) }\end{array}$ & filipe & & M & G & a & $\mathrm{R}$ \\
\hline Myiobius sp. & & & M & G & & $\mathrm{O}$ \\
\hline Hirundinea ferruginea (Gmelin, 1788) & gibão-de-couro & & $\mathrm{C}$ & G & a & $\mathrm{R}$ \\
\hline Lathrotriccus euleri (Cabanis, 1868) & enferrujado & & M & $\mathrm{G} / \mathrm{F}$ & a & $\mathrm{E}$ \\
\hline Knipolegus lophotes Boie, 1828 & maria-preta-de-penacho & & $\mathrm{C}$ & & & $\mathrm{E}$ \\
\hline Knipolegus nigerrimus (Vieillot, 1818) & $\begin{array}{l}\text { maria-preta-de-garganta- } \\
\text { vermelha }\end{array}$ & ATL & $\mathrm{C}$ & & & $\mathrm{O}$ \\
\hline
\end{tabular}


Anexo 1. Continuação...

\begin{tabular}{|c|c|c|c|c|c|c|}
\hline Famílias e espécies & Nome popular & $\begin{array}{l}\text { Endemismo/ } \\
\text { Conservação }\end{array}$ & Ambiente & Registro & Migração & Freqüiência \\
\hline Fluvicola nengeta (Linnaeus, 1766) & lavadeira-mascarada & & $\mathrm{D}$ & & & $\mathrm{R}$ \\
\hline Colonia colonus (Vieillot, 1818) & viuvinha & & M & G & & $\mathrm{R}$ \\
\hline Machetornis rixosa (Vieillot, 1819) & suiriri-cavaleiro & & M & & a & $\mathrm{O}$ \\
\hline Legatus leucophaius (Vieillot, 1818) & bem-te-vi-pirata & & M & G & $\mathrm{np} / \mathrm{a}$ & $\mathrm{R}$ \\
\hline Myiozetetes similis (Spix, 1825) & $\begin{array}{l}\text { bentevizinho-de-penacho- } \\
\text { vermelho }\end{array}$ & & $\mathrm{D}$ & & & $\mathrm{E}$ \\
\hline Pitangus sulphuratus (Linnaeus, 1766) & bem-te-vi & & $\mathrm{D}$ & G & a & $\mathrm{E}$ \\
\hline $\begin{array}{l}\text { Myiodynastes maculatus } \\
\text { (Statius Muller, 1776) }\end{array}$ & bem-te-vi-rajado & & M & G & a & $\mathrm{R}$ \\
\hline Megarynchus pitangua (Linnaeus, 1766) & neinei & & M & $\mathrm{G}$ & a & $\mathrm{C}$ \\
\hline Tyrannus melancholicus Vieillot, 1819 & suiriri & & $\mathrm{D}$ & G & $\mathrm{np} / \mathrm{a}$ & $\mathrm{E}$ \\
\hline Sirystes sibilator (Vieillot, 1818) & gritador & & M & $\mathrm{G}$ & $\mathrm{a}$ & $\mathrm{R}$ \\
\hline $\begin{array}{l}\text { Myiarchus swainsoni } \\
\text { Cabanis \& Heine, } 1859\end{array}$ & irré & & $\mathrm{M} / \mathrm{D}$ & G & a & $\mathrm{R}$ \\
\hline Myiarchus ferox (Gmelin, 1789) & maria-cavaleira & & $\mathrm{M} / \mathrm{D} / \mathrm{C}$ & $\mathrm{G}$ & & $\mathrm{C}$ \\
\hline \multicolumn{7}{|l|}{ Pipridae Rafinesque, 1815} \\
\hline Neopelma pallescens (Lafresnaye, 1853) & fruxu-do-cerradão & & M & $\mathrm{G} / \mathrm{F}$ & & $\mathrm{R}$ \\
\hline Ilicura militaris (Shaw \& Nodder, 1809) & tangarazinho & ATL & M & $\mathrm{G} / \mathrm{F}$ & & A \\
\hline $\begin{array}{l}\text { Chiroxiphia caudata } \\
\text { (Shaw \& Nodder, 1793) }\end{array}$ & tangará & ATL & M & $\mathrm{G} / \mathrm{F}$ & & A \\
\hline \multicolumn{7}{|l|}{ Tityridae Gray, 1840} \\
\hline Schiffornis virescens (Lafresnaye, 1838) & flautim & ATL & M & $\mathrm{G}$ & & $\mathrm{E}$ \\
\hline \multicolumn{7}{|l|}{ Vireonidae Swainson, 1837 (16) } \\
\hline Cyclarhis gujanensis (Gmelin, 1789) & pitiguari & & M & G & & $\mathrm{C}$ \\
\hline Vireo olivaceus (Linnaeus, 1766) & juruviara & & M & $\mathrm{G} / \mathrm{F}$ & $\mathrm{n} / \mathrm{a}$ & $\mathrm{E}$ \\
\hline $\begin{array}{l}\text { Hylophilus amaurocephalus } \\
\text { (Nordmann, 1835) }\end{array}$ & vite-vite-de-olho-cinza & & M & $\mathrm{G} / \mathrm{F}$ & & $\mathrm{C}$ \\
\hline \multicolumn{7}{|l|}{ Corvidae Leach, 1820} \\
\hline $\begin{array}{l}\text { Cyanocorax cristatellus } \\
\text { (Temminck, 1823) }\end{array}$ & gralha-do-campo & $\mathrm{CE}$ & $\mathrm{C}$ & G & & $\mathrm{R}$ \\
\hline \multicolumn{7}{|l|}{ Hirundinidae Rafinesque, 1815 (16) } \\
\hline Pygochelidon cyanoleuca (Vieillot, 1817) & andorinha-pequena-de-casa & & $\mathrm{M} / \mathrm{D} / \mathrm{C}$ & $\mathrm{G}$ & a & $\mathrm{C}$ \\
\hline Stelgidopteryx ruficollis (Vieillot, 1817) & andorinha-serradora & & $\mathrm{D}$ & & a & $\mathrm{O}$ \\
\hline Progne tapera (Vieillot, 1817) & andorinha-do-campo & & $\mathrm{D}$ & & a & $\mathrm{R}$ \\
\hline Progne chalybea (Gmelin, 1789) & $\begin{array}{l}\text { andorinha-doméstica- } \\
\text { grande }\end{array}$ & & $\mathrm{D}$ & & $\mathrm{n} / \mathrm{a}$ & $\mathrm{O}$ \\
\hline \multicolumn{7}{|l|}{ Troglodytidae Swainson, 1831 (18) } \\
\hline Troglodytes musculus Naumann, 1823 & corruíra & & $\mathrm{D}$ & $\mathrm{G}$ & & $\mathrm{C}$ \\
\hline \multicolumn{7}{|l|}{ Turdidae Rafinesque, 1815 (17) } \\
\hline Turdus subalaris (Seebohm, 1887) & sabiá-ferreiro & & M & & a & $\mathrm{O}$ \\
\hline Turdus rufiventris Vieillot, 1818 & sabiá-laranjeira & & $\mathrm{M} / \mathrm{D}$ & $\mathrm{G} / \mathrm{F}$ & & A \\
\hline Turdus amaurochalinus Cabanis, 1850 & sabiá-poca & & $\mathrm{M} / \mathrm{D}$ & $\mathrm{G}$ & a & $\mathrm{R}$ \\
\hline Turdus leucomelas Vieillot, 1818 & sabiá-barranco & & $\mathrm{M} / \mathrm{D}$ & G & & A \\
\hline \multicolumn{7}{|l|}{ Mimidae Bonaparte, 1853} \\
\hline Mimus saturninus (Lichtenstein, 1823) & sabiá-do-campo & & $\mathrm{D} / \mathrm{C}$ & $\mathrm{G}$ & & $\mathrm{R}$ \\
\hline \multicolumn{7}{|l|}{ Coerebidae d'Orbigny \& Lafresnaye, 1838} \\
\hline Coereba flaveola (Linnaeus, 1758) & cambacica & & $\mathrm{M} / \mathrm{D} / \mathrm{C}$ & $\mathrm{G} / \mathrm{F}$ & & A \\
\hline \multicolumn{7}{|l|}{ Thraupidae Cabanis, 1847 (85) } \\
\hline Trichothraupis melanops (Vieillot, 1818) & tiê-de-topete & & M & $\mathrm{G} / \mathrm{F}$ & & $\mathrm{E}$ \\
\hline Piranga flava (Vieillot, 1822) & sanhaçu-de-fogo & & $\mathrm{D}$ & & $\mathrm{np} / \mathrm{a}$ & $\mathrm{O}$ \\
\hline
\end{tabular}


Anexo 1. Continuação...

\begin{tabular}{|c|c|c|c|c|c|c|}
\hline Famílias e espécies & Nome popular & $\begin{array}{l}\text { Endemismo/ } \\
\text { Conservação }\end{array}$ & Ambiente & Registro & Migração & Freqüência \\
\hline Tachyphonus coronatus (Vieillot, 1822) & tiê-preto & ATL & M & $\mathrm{G} / \mathrm{F}$ & & $\mathrm{E}$ \\
\hline Thraupis sayaca (Linnaeus, 1766) & sanhaçu-cinzento & & $\mathrm{M} / \mathrm{D}$ & G & a & $\mathrm{C}$ \\
\hline Thraupis ornata (Sparrman, 1789) & $\begin{array}{c}\text { sanhaçu-de-encontro- } \\
\text { amarelo }\end{array}$ & ATL & M & & & $\mathrm{O}$ \\
\hline Pipraeidea melanonota (Vieillot, 1819) & saíra-viúva & & $\mathrm{C}$ & & & $\mathrm{R}$ \\
\hline Tangara cyanoventris (Vieillot, 1819) & saíra-douradinha & ATL & $\mathrm{M} / \mathrm{D}$ & $\mathrm{G} / \mathrm{F}$ & & A \\
\hline Tangara cayana (Linnaeus, 1766) & saíra-amarela & & $\mathrm{C} / \mathrm{D}$ & $\mathrm{G} / \mathrm{F}$ & & $\mathrm{E}$ \\
\hline Tersina viridis (Illiger, 1811) & saí-andorinha & & M & & a & $\mathrm{R}$ \\
\hline Dacnis cayana (Linnaeus, 1766) & saí-azul & & $\mathrm{M} / \mathrm{D}$ & & & $\mathrm{E}$ \\
\hline Hemithraupis ruficapilla (Vieillot, 1818) & saíra-ferrugem & ATL & $\mathrm{M} / \mathrm{D}$ & $\mathrm{G} / \mathrm{F}$ & & A \\
\hline \multicolumn{7}{|l|}{ Emberizidae Vigors, 1825 (74) } \\
\hline $\begin{array}{l}\text { Zonotrichia capensis } \\
\text { (Statius Muller, 1776) }\end{array}$ & tico-tico & & $\mathrm{C}$ & G & a & $\mathrm{E}$ \\
\hline Haplospiza unicolor Cabanis, 1851 & cigarra-bambu & ATL & M & G & $\mathrm{mr}$ & $\mathrm{O}$ \\
\hline Poospiza cinerea Bonaparte, 1850 & capacetinho-do-oco-do-pau & $\mathrm{CE}, \mathrm{gv}, \mathrm{rv}$ & $\mathrm{C}$ & & & $\mathrm{O}$ \\
\hline Sicalis citrina Pelzeln, 1870 & canário-rasteiro & & $\mathrm{F}$ & G & & $\mathrm{E}$ \\
\hline Embernagra longicauda Strickland, 1844 & rabo-mole-da-serra & TM, gq & $\mathrm{F}$ & G & & A \\
\hline Volatinia jacarina (Linnaeus, 1766) & tiziu & & $\mathrm{D}$ & $\mathrm{G} / \mathrm{F}$ & a & $\mathrm{R}$ \\
\hline Sporophila nigricollis (Vieillot, 1823) & baiano & & $\mathrm{D}$ & $\mathrm{G} / \mathrm{F}$ & & $\mathrm{R}$ \\
\hline Sporophila caerulescens (Vieillot, 1823) & coleirinho & & $\mathrm{D}$ & & a & $\mathrm{O}$ \\
\hline Arremon flavirostris Swainson, 1838 & tico-tico-de-bico-amarelo & & M & $\mathrm{G} / \mathrm{F}$ & & $\mathrm{R}$ \\
\hline Coryphospingus pileatus (Wied, 1821) & tico-tico-rei-cinza & & $\mathrm{M} / \mathrm{C} / \mathrm{F}$ & $\mathrm{G} / \mathrm{F}$ & & $\mathrm{R}$ \\
\hline \multicolumn{7}{|l|}{ Cardinalidae Ridgway, 1901} \\
\hline $\begin{array}{l}\text { Saltator similis } \\
\text { d'Orbigny \& Lafresnaye, } 1837\end{array}$ & trinca-ferro-verdadeiro & & $\mathrm{C}$ & G & & $\mathrm{E}$ \\
\hline \multicolumn{7}{|c|}{ Parulidae Wetmore, Friedmann, Lincoln, Miller, Peters, van Rossem, Van Tyne \& Zimmer 1947 (22) } \\
\hline Geothlypis aequinoctialis (Gmelin, 1789) & pia-cobra & & $\mathrm{C}$ & G & a & $\mathrm{R}$ \\
\hline Basileuterus hypoleucus Bonaparte, 1830 & pula-pula-de-barriga-branca & & M & $\mathrm{G} / \mathrm{F}$ & & A \\
\hline Basileuterus flaveolus (Baird, 1865) & canário-do-mato & & M & $\mathrm{G} / \mathrm{F}$ & & $\mathrm{C}$ \\
\hline $\begin{array}{l}\text { Basileuterus leucoblepharus } \\
\text { (Vieillot, 1817) }\end{array}$ & pula-pula-assobiador & ATL & M & G & & $\mathrm{R}$ \\
\hline \multicolumn{7}{|l|}{ Icteridae Vigors, 1825} \\
\hline Psarocolius decumanus (Pallas, 1769) & japu & & M & $\mathrm{G}$ & & $\mathrm{O}$ \\
\hline Gnorimopsar chopi (Vieillot, 1819) & graúna & & $\mathrm{D}$ & & & $\mathrm{O}$ \\
\hline Carduelis magellanica (Vieillot, 1805) & pintassilgo & & $\mathrm{C}$ & G & & $\mathrm{O}$ \\
\hline \multicolumn{7}{|l|}{ Fringillidae Leach, 1820} \\
\hline Euphonia chlorotica (Linnaeus, 1766) & fim-fim & & $\mathrm{M} / \mathrm{D}$ & G & & $\mathrm{C}$ \\
\hline \multicolumn{7}{|l|}{ Estrildidae Bonaparte, 1850} \\
\hline Estrilda astrild (Linnaeus, 1758) & bico-de-lacre & & $\mathrm{D}$ & & & $\mathrm{R}$ \\
\hline \multicolumn{7}{|l|}{ Passeridae Rafinesque, 1815} \\
\hline Passer domesticus (Linnaeus, 1758) & pardal & & $\mathrm{D}$ & & & $\mathrm{O}$ \\
\hline
\end{tabular}


Anexo 2. Distribuição e situação de conservação das espécies ameaçadas e/ou endêmicas em seis Unidades de Conservação do Quadrilátero Ferrífero. Conservação: (gc) criticamente em perigo, (gp) em perigo, (gv) vulnerável e (gq) quase-ameaçado de extinção no mundo (BirdLife International 2008); (rc) criticamente em perigo (rp), em perigo e (rv) vulnerável à extinção em Minas Gerais (Machado et al. 1998). Endemismo: (1) endêmica da Mata Atlântica, segundo Stotz et al. (1996); (2) endêmica do Cerrado e (3) endêmica dos topos de montanha do sudeste do Brasil, segundo Silva (2002) e Vasconcelos et al. (2003a). Classificação taxonômica segundo CBRO (2008).

Annex 2. Distribution and conservation status of threatened and/or endemic species at six Iron Quadrangle Conservation Units areas in Minas Gerais State, Brazil. Conservation: globally (gc) critically endangered, (gp) endangered, (gv) vulnerable, and (gq) near-threatened (BirdLife International 2008); regionally (at Minas Gerais state) (rc) critically endangered, (rp) endangered, and (rv) vulnerable (Machado et al. 1998). Endemism: (1) Altantic Forest endemics, according to Stotz et al. (1996); (2) Cerrado endemics, and (3) top mountain of southeastern Brazil endemics, according to Silva (2002), and Vasconcelos et al. (2003a). Taxonomic classification according to CBRO (2008).

\begin{tabular}{|c|c|c|c|c|c|c|c|}
\hline Espécies & $\begin{array}{c}\text { Endemismo e } \\
\text { conservação }\end{array}$ & MSP & PEI & EPDA Peti & $\begin{array}{l}\text { RPPN } \\
\text { Caraça }\end{array}$ & PPSC & PESRM \\
\hline Tinamus solitarius & $1, \mathrm{gq}, \mathrm{rc}$ & & & $\mathrm{x} * *$ & & & $\mathrm{x} * *$ \\
\hline crypturellus noctivagus & $1, \mathrm{gq}, \mathrm{rc}$ & & & & $\mathrm{x} * ?$ & & \\
\hline Mergus octosetaceus & $2, \mathrm{gc}, \mathrm{rc}$ & & $\mathrm{x}$ & & & & \\
\hline Penelope obscura & $\mathrm{rv}$ & & $\mathrm{x}$ & $\mathrm{x}$ & $\mathrm{x}$ & & \\
\hline Crax blumenbachii & $1, \mathrm{gp}, \mathrm{rc}$ & & & $\mathrm{x} * *$ & & & $\mathrm{x} * *$ \\
\hline Odontophorus capueira & $1, \mathrm{rv}$ & & & & $\mathrm{x}$ & & $\mathrm{x} * *$ \\
\hline Cathartes burrovianus & 1 & & & $\mathrm{x}$ & & & \\
\hline Leucopternis polionotus & $1, \mathrm{gq} \mathrm{rp}$ & & & & $\mathrm{x}$ & & \\
\hline Harpyhaliaetus coronatus & $\mathrm{gp}, \mathrm{rp}$ & & & & & & $\mathrm{x}$ \\
\hline Spizaetus tyrannus & $\mathrm{rp}$ & & & & $\mathrm{x} *$ & & \\
\hline Aramides saracura & 1 & $\mathrm{x}$ & $\mathrm{x}$ & $\mathrm{x}$ & $\mathrm{x}$ & & \\
\hline Amazona vinacea & $1, \mathrm{gv}, \mathrm{rp}$ & & $\mathrm{x}^{*}$ & & & & \\
\hline Pyrrhura frontalis & 1 & & $\mathrm{x}^{*}$ & & $\mathrm{x}$ & & \\
\hline Primolius maracana & $\mathrm{gq}$ & & $\mathrm{x}^{*}$ & & $\mathrm{x}$ & & \\
\hline Pulsatrix koeniswaldiana & 1 & & $\mathrm{x}^{*}$ & & $\mathrm{x}$ & & \\
\hline Strix hylophila & $1, \mathrm{gq}$ & & $\mathrm{x}$ & & & & \\
\hline Macropsalis forcipata & $1, \mathrm{rv}$ & & $\mathrm{x}$ & & & & \\
\hline Phaethornis eurynome & 1 & & $\mathrm{x}$ & & $\mathrm{x}$ & & \\
\hline Aphantochroa cirrochloris & 1 & & & $\mathrm{x}$ & & & \\
\hline Florisuga fusca & 1 & $\mathrm{x}$ & $\mathrm{x}$ & $\mathrm{x}$ & $\mathrm{x}$ & $\mathrm{x}$ & \\
\hline Thalurania glaucopis & 1 & $\mathrm{x}$ & $\mathrm{x}$ & $\mathrm{x}$ & $\mathrm{x}$ & $\mathrm{x}$ & $\mathrm{x}$ \\
\hline Leucochloris albicollis & 1 & & $\mathrm{x}$ & & $\mathrm{x}$ & & \\
\hline Clytolaema rubricauda & 1 & & $\mathrm{x}$ & & $\mathrm{x}$ & & \\
\hline Augastes scutatus & $2, \mathrm{gq}$ & & $\mathrm{x}$ & $\mathrm{x}$ & $\mathrm{x}$ & & $\mathrm{x}$ \\
\hline Trogon surrucura & 1 & $\mathrm{x}$ & $\mathrm{x}$ & $\mathrm{x}$ & $\mathrm{x}$ & & \\
\hline Baryphthengus ruficapillus & 1 & & & $\mathrm{x} *$ & & & \\
\hline Malacoptila striata & 1 & & & $\mathrm{x}$ & $\mathrm{x}$ & & \\
\hline Ramphastos dicolorus & 1 & & & & $\mathrm{x}$ & & \\
\hline Melanerpes flavifrons & 1 & & & & $\mathrm{x} * ?$ & & \\
\hline Veniliornis maculifrons & 1 & & $\mathrm{x}$ & & & & \\
\hline Piculus aurulentus & $1, \mathrm{gq}$ & & & & $\mathrm{x}$ & & \\
\hline Campephilus robustus & $1, \mathrm{rp}$ & $\mathrm{x}$ & & & & & \\
\hline Melanopareia torquata & 2 & $\mathrm{x}$ & & & & $\mathrm{x}$ & $\mathrm{x}$ \\
\hline Mackenziaena leachii & 1 & $\mathrm{x}$ & $\mathrm{x}$ & $\mathrm{x}$ & $\mathrm{x}$ & & $\mathrm{x}$ \\
\hline Mackenziaena severa & 1 & & $\mathrm{x}$ & $\mathrm{x} *$ & $\mathrm{x}$ & & \\
\hline Formicivora serrana & 1 & $\mathrm{x}$ & $\mathrm{x}^{*}$ & $\mathrm{x}$ & $\mathrm{x}$ & & \\
\hline Drymophila ferruginea & 1 & $\mathrm{x}$ & $\mathrm{x}$ & $\mathrm{x} *$ & $\mathrm{x}$ & & $\mathrm{x}$ \\
\hline Drymophila rubricollis & 1 & & & & $\mathrm{x}$ & & \\
\hline Drymophila ochropyga & $1, \mathrm{gq}$ & & $\mathrm{x}$ & $\mathrm{x}$ & $\mathrm{x}$ & & \\
\hline Drymophila malura & 1 & & $\mathrm{x}$ & & $\mathrm{x}$ & & \\
\hline Pyriglena leucoptera & 1 & $\mathrm{x}$ & $\mathrm{x}$ & $\mathrm{x}$ & $\mathrm{x}$ & $\mathrm{x}$ & $\mathrm{x}$ \\
\hline Myrmeciza loricata & 1 & & $\mathrm{x}$ & $\mathrm{x}$ & $\mathrm{x}$ & & \\
\hline Conopophaga lineata & 1 & $\mathrm{x}$ & $\mathrm{x}$ & $\mathrm{x}$ & $\mathrm{x}$ & & $\mathrm{x}$ \\
\hline Hylopezus nattereri & 1 & & $\mathrm{x}$ & & $\mathrm{x}$ & & \\
\hline Psilorhamphus guttatus & $1, \mathrm{gq}$ & & $\mathrm{x}^{*}$ & & & & \\
\hline Scytalopus speluncae & 1 & & $\mathrm{x}$ & & & & \\
\hline
\end{tabular}


Anexo 2. Continuação...

\begin{tabular}{|c|c|c|c|c|c|c|c|}
\hline Espécies & $\begin{array}{c}\text { Endemismo e } \\
\text { conservação }\end{array}$ & MSP & PEI & EPDA Peti & $\begin{array}{c}\text { RPPN } \\
\text { Caraça }\end{array}$ & PPSC & PESRM \\
\hline Scytalopus indigoticus & $1, \mathrm{gq}$ & $\mathrm{x}$ & $\mathrm{x}$ & $\mathrm{x}$ & $\mathrm{x}$ & & \\
\hline Chamaeza meruloides & 1 & & $\mathrm{x}^{*}$ & & $\mathrm{x}$ & & \\
\hline Sclerurus scansor & 1 & & $\mathrm{x}$ & & $\mathrm{x}$ & & \\
\hline Xiphorhynchus fuscus & 1 & $\mathrm{x}$ & $\mathrm{x}$ & $\mathrm{x}$ & $\mathrm{x}$ & & \\
\hline Lepidocolaptes squamatus & 1 & & $\mathrm{x}$ & $\mathrm{x}$ & $\mathrm{x}$ & & $\mathrm{x}$ \\
\hline Campylorhamphus falcularius & 1 & & $\mathrm{x}$ & & $\mathrm{x}$ & & \\
\hline Oreophylax moreirae & 1 & & & & $\mathrm{x}$ & & \\
\hline Synallaxis ruficapilla & 1 & $\mathrm{x}$ & $\mathrm{x}$ & $\mathrm{x}$ & $\mathrm{x}$ & & $\mathrm{x}$ \\
\hline Cranioleuca pallida & 1 & & $\mathrm{x}$ & $\mathrm{x} *$ & $\mathrm{x}$ & & \\
\hline Phacellodomus erythrophthalmus & 1 & & $\mathrm{x}$ & $\mathrm{x} *$ & $\mathrm{x}$ & $\mathrm{x}$ & \\
\hline Philydor atricapillus & 1 & & & & $\mathrm{x} * ?$ & & \\
\hline Anabazenops fuscus & 1 & & $\mathrm{x}$ & & $\mathrm{x} *$ & & \\
\hline Automolus leucophthalmus & 1 & $\mathrm{x}$ & $\mathrm{x}$ & $\mathrm{x}$ & $\mathrm{x}$ & & $\mathrm{x}$ \\
\hline Mionectes rufiventris & 1 & $\mathrm{x}$ & $\mathrm{x}$ & $\mathrm{x}$ & $\mathrm{x}$ & & $\mathrm{x}$ \\
\hline Hemitriccus diops & 1 & & $\mathrm{x}$ & & $\mathrm{x}$ & & \\
\hline Hemitriccus nidipendulus & 1 & $\mathrm{x}$ & $\mathrm{x}$ & $\mathrm{x}$ & $\mathrm{x}$ & & \\
\hline Myiornis auricularis & 1 & $\mathrm{x}$ & $\mathrm{x}$ & $\mathrm{x}$ & $\mathrm{x} *$ & & \\
\hline Todirostrum poliocephalum & 1 & $\mathrm{x}$ & $\mathrm{x}$ & $\mathrm{x}$ & $\mathrm{x}$ & & $\mathrm{x}$ \\
\hline Phyllomyias virescens & 1 & & $\mathrm{x}^{*}$ & & & & \\
\hline Polystictus superciliaris & 3 & & $\mathrm{x}^{*}$ & & $\mathrm{x}$ & $\mathrm{x}$ & $\mathrm{x}$ \\
\hline Phylloscartes eximius & $1, \mathrm{gq}$ & & $\mathrm{x}$ & & & & \\
\hline Knipolegus nigerrimus & 1 & $\mathrm{x}$ & $\mathrm{x}$ & $\mathrm{x}$ & $\mathrm{x}$ & $\mathrm{x}$ & $\mathrm{x}$ \\
\hline Muscipipra vetula & 1 & & $\mathrm{x}$ & $\mathrm{x} *$ & $\mathrm{x}$ & & \\
\hline Attila rufus & 1 & & & & $\mathrm{x} * ?$ & & \\
\hline Phibalura flavirostris & $\mathrm{gq}, \mathrm{rv}$ & & $\mathrm{x}$ & & $\mathrm{x}$ & & \\
\hline Lipaugus lanioides & $1, \mathrm{gq}, \mathrm{rv}$ & & $\mathrm{x}^{*}$ & & $\mathrm{x}$ & & \\
\hline Pyroderus scutatus & $1, \mathrm{rv}$ & & $\mathrm{x}$ & $\mathrm{x}$ & $\mathrm{x}$ & & \\
\hline Neopelma aurifrons & $1, \mathrm{gv}$ & & & $\mathrm{x} \#$ & & & \\
\hline Neopelma chrysolophum & 1 & & & $\mathrm{x}$ & $\mathrm{x}$ & & \\
\hline Ilicura militaris & 1 & $\mathrm{x}$ & $\mathrm{x}$ & $\mathrm{x}$ & $\mathrm{x}$ & $\mathrm{x}$ & $\mathrm{x}$ \\
\hline Antilophia galeata & 2 & & & & & & $\mathrm{x}$ \\
\hline Chiroxiphia caudata & 1 & $\mathrm{x}$ & $\mathrm{x}$ & $\mathrm{x}$ & $\mathrm{x}$ & $\mathrm{x}$ & $\mathrm{x}$ \\
\hline Schiffornis virescens & 1 & $\mathrm{x}$ & $\mathrm{x}$ & $\mathrm{x}$ & $\mathrm{x}$ & & \\
\hline Laniisoma elegans & $\mathrm{rv}$ & & & & $\mathrm{x}$ & & \\
\hline Hylophilus poicilotis & 1 & & $\mathrm{x} * ?$ & & $\mathrm{x} * ?$ & & \\
\hline Cyanocorax cristatellus & 2 & $\mathrm{x}$ & & $\mathrm{x} *$ & $\mathrm{x}$ & $\mathrm{x}$ & $\mathrm{x}$ \\
\hline Tachyphonus coronatus & 1 & $\mathrm{x}$ & $\mathrm{x}$ & $\mathrm{x} * *$ & $\mathrm{x}$ & $\mathrm{x}$ & $\mathrm{x}$ \\
\hline Thraupis ornata & 1 & $\mathrm{x}$ & $\mathrm{x}$ & $\mathrm{x} * *$ & $\mathrm{x}$ & & \\
\hline Euphonia cyanocephala & 1 & & & $\mathrm{x} *$ & $\mathrm{x}$ & & \\
\hline Tangara seledon & 1 & & & & $\mathrm{x} *$ & & \\
\hline Tangara desmaresti & 1 & & $\mathrm{x}$ & $\mathrm{x} *$ & $\mathrm{x}$ & & \\
\hline Tangara cyanoventris & 1 & $\mathrm{x}$ & $\mathrm{x}$ & $\mathrm{x}$ & $\mathrm{x}$ & $\mathrm{x}$ & $\mathrm{x}$ \\
\hline Hemithraupis ruficapilla & 1 & $\mathrm{x}$ & $\mathrm{x}$ & $\mathrm{x}$ & $\mathrm{x}$ & $\mathrm{x}$ & $\mathrm{x}$ \\
\hline Porphyrospiza caerulescens & $2, \mathrm{gq}$ & & & & $\mathrm{x} *$ & $\mathrm{x}$ & $\mathrm{X}$ \\
\hline Haplospiza unicolor & 1 & $\mathrm{x}$ & $\mathrm{x}^{*}$ & & $\mathrm{x}$ & & \\
\hline Poospiza cinerea & $2, \mathrm{gv}, \mathrm{rv}$ & $\mathrm{x}$ & & & $\mathrm{x} *$ & $\mathrm{x}$ & \\
\hline Sicalis flaveola & rv & & $\mathrm{x}^{*}$ & $\mathrm{x} * *$ & $\mathrm{x}$ & & \\
\hline Embernagra longicauda & $3, \mathrm{gq}$ & $\mathrm{x}$ & $\mathrm{x}$ & $\mathrm{x} *$ & $\mathrm{x}$ & $\mathrm{x}$ & $\mathrm{x}$ \\
\hline Saltator atricollis & 2 & & & & $\mathrm{x}$ & & \\
\hline Basileuterus leucoblepharus & 1 & $\mathrm{x}$ & $\mathrm{x}$ & & $\mathrm{x}$ & & $\mathrm{x}$ \\
\hline
\end{tabular}

(*) Espécies observadas por Carnevalli em EPDA Peti em 1989, em RPPN Caraça em 1980 e em PEI por Mattos na década de 80 e não observados nos levantamentos posteriores. (?) Espécies que podem ter sido registradas por erro de identificação. (\#) registrada por Carnevalli em Peti em 1989, antes da revisão da espécie por Whitney et al. (1995). (**) Espécies participantes de programas de reintrodução. (?), (\#) e (**) não foram utilizadas nos cálculos. 